\title{
Targeting matrix metalloproteinases with novel diazepine substituted cinnamic acid derivatives: design, synthesis, in vitro and in silico studies
}

\author{
Dharmender Rathee ${ }^{1}$, Viney Lather ${ }^{2}$, Ajmer Singh Grewal $^{3}$ and Harish Dureja ${ }^{*^{*}}$
}

\begin{abstract}
Lung cancer is the notable cause of cancer associated deaths worldwide. Recent studies revealed that the expression of matrix metalloproteinases (MMPs) is extremely high in lung tumors compared with non-malignant lung tissue. MMPs (-2 and -9) play an important part in tumor development and angiogenesis, which suggests that creating potent MMP-2 and -9 inhibitors, should be an important goal in lung cancer therapy. In the present study, an effort has been made to develop new anti-metastatic and anti-invasive agents, wherein a series of novel diazepine substituted cinnamic acid derivatives were designed, synthesized and assayed for their inhibitory activities on MMP-2 and MMP-9. These derivatives were prepared via microwave assisted reaction of tert-butyl (3-cinnamamidopropyl) carbamate derivatives mixed with 2,3-dibromopropanoic acid and potassium carbonate was added to obtain 4-(tertbutoxycarbonyl)-1-cinnamoyl-1,4-diazepane-2-carboxylic acid derivatives. The newly synthesized compounds were characterized by IR, NMR and mass spectroscopy. All the tested compounds showed good to excellent cytotoxic potential against A549 human lung cancer cells. The active compounds displaying good activity were further examined for the inhibitory activity against MMPs (-2 and -9$)$. In addition, the structure and anticancer activity relationship were further supported by in silico docking studies of the active compounds against MMP-2 and MMP-9.
\end{abstract}

Keywords: Targeting, MMP-2, MMP-9, Diazepine, Cinnamic acid, Molecular docking

\section{Introduction}

Malignant properties of lung polyp cells, such as metastasis, tissue invasion, irregular tumor growth, tissue remodeling and inflammation, are linked with reformed proteolysis [3, 22]. Matrix metalloproteinases (MMPs) exemplify the most significant group of proteinases, which gets activated directly by degrading the extracellular matrix (ECM) and/or other secreted proteins of the lungs. Conversely, by altering the properties of the cleaved proteins in the alveolar space, MMPs function independently of their proteolytic activity [27]. MMPs are zinc-dependent endopeptidases [5] commonly known as

\footnotetext{
${ }^{*}$ Correspondence: harishdureja@gmail.com

1 Department of Pharmaceutical Sciences, Maharshi Dayanand University, Rohtak, Haryana 124001, India

Full list of author information is available at the end of the article
}

matrixins, which play a special role during tissue remodeling and organ development $[18,34]$. Aberration in the expression of MMP is associated with a variety of diseases from respiratory to autoimmune disorder and even cancer, particularly lung cancer. MMPs are known to influence lung cancer metastatic properties and involved several signalling pathways [16]. MMP-2 and -9; gelatinases, are very closely associated with the metastatic properties of lung cancer [39], which suggests that creating potent MMP-2 and MMP-9 inhibitors should be an important goal in lung cancer therapy [31].

In the current study, we have used fragment linking and structure based approaches for the design of diazepine substituted cinnamic acid molecule as it involves two (or more) fragments, and extended $\mathrm{P}^{\prime}$ group. The fragments which are active against one receptor are joined together to give a higher affinity molecule and the cinnamic acid 
amides with extended $\mathrm{P} 1^{\prime}$ group could further increase the activity. In SAR studies a standard nomenclature Pn, ... P1, P2, P3 etc. is used to designate amino acid residues of a peptide substrate (Example of P1 group such as branched alkanes and cycloalkanes) $[1,10]$. Various reports have shown diazepine and caffeic acid (hydroxycinnamic acid) derivatives as the active moieties against MMPs [14, 24, 28, 29, 33, 36]. Several modified caffeic acid amides have more steady features [25]. These results encouraged us to design and synthesize a novel series of diazepine substituted cinnamic acid derivatives to explore their inhibitory activity on MMP-2 and MMP-9 (Fig. 1) and their structure-activity relationship (SAR) analysis.

\section{Materials and methods Chemicals}

All the chemicals were purchased from Thermo Fisher Scientific and were used as such for the experiments. Melting points were determined using Veego VMP-D melting point apparatus. Thin layer chromatography (Merck silica gel-G) was used to monitor the reaction progress. ${ }^{1} \mathrm{H}$ and ${ }^{13} \mathrm{C}$ NMR spectra were recorded by Bruker Avance II $300 \mathrm{MHz}$ NMR spectrometer using DMSO-d6 as solvent and are expressed in parts per million $(\delta, \mathrm{ppm})$ downfield from tetramethylsilane (internal standard). NMR data is given as multiplicity (s, singlet; $d$, doublet; $\mathrm{t}$, triplet; $\mathrm{m}$, multiplet) and number of protons. Infrared (IR) spectra were recorded by $\mathrm{KBr}$ disc method on a Shimadzu IR affinity FTIR spectrophotometer. The wave number is given in $\mathrm{cm}^{-1}$. Mass spectra were taken on Waters, Q-TOF Micromass spectrometer (ESI-MS).

\section{Synthesis of diazepine substituted cinnamic acid derivatives}

Benzaldehyde derivative (1.0 molar eq.) and malonic acid (2.2 molar eq.) were added to $50 \mathrm{~mL}$ of dry pyridine, containing ( 0.015 molar eq.) of aniline, to form a solution.
This solution was allowed to stand overnight, followed by heating for $3 \mathrm{~h}$ at $55^{\circ} \mathrm{C}$ in order to remove carbon dioxide. Reaction mixture was then poured into the mixture of $60 \mathrm{~mL}$ of concentrated hydrochloric acid and $100 \mathrm{~g}$ of chopped ice. The acid precipitated immediately and then allowed to stand for few minutes for complete separation. The filtration was done followed by washing of product with $10 \mathrm{~mL}$ of $5 \%$ hydrochloric acid and then with two portions of $10 \mathrm{~mL}$ water. At the end, drying of residue was carried out. Cinnamic acid derivatives obtained above (1.0 molar eq.) were refluxed with thionyl chloride (1.1 molar eq.) for $4 \mathrm{~h}$ in order to obtain the corresponding acid chlorides. Henceforth, the acid chlorides obtained above were refluxed with tert-butyl (3-aminopropyl)carbamate (1:1) for $4 \mathrm{~h}$ and respective tert-butyl (3-cinnamamidopropyl)carbamate derivatives were synthesized. Respective tert-butyl (3-cinnamamidopropyl)carbamate derivatives $(1.0 \mathrm{mmol})$ were mixed with 2,3-dibromopropanoic acid $(1.1 \mathrm{mmol})$ and potassium carbonate (1.1 mmol) was added to obtain 4-(tert-butoxycarbonyl)1-cinnamoyl-1,4-diazepane-2-carboxylic acid derivatives. This step was performed under microwave irradiation at temperature of $120^{\circ} \mathrm{C}$ and power at $90 \mathrm{~W}$ for $20 \mathrm{~min}$. The extraction of organic portion was carried out with ethyl acetate. The solvent was removed and the product was recrystallized [11]. Then, to a solution, of synthesized 4-(tert-butoxycarbonyl)-1-cinnamoyl-1,4-diazepane2-carboxylic acid derivatives (1.0 molar eq.) and methanol (36.72 molar eq.), thionyl chloride (5.0 molar eq.) was added dropwise (at room temperature). After that, stirring was performed overnight to synthesize 1-tert-butyl 3-methyl 4-cinnamoyl-1,4-diazepane-1,3-dicarboxylate derivatives and dried by the agency of rotary evaporator. Various acyl and aryl acid chlorides were refluxed with 1-tert-butyl 3-methyl 4-cinnamoyl-1,4-diazepane1,3-dicarboxylate derivatives (1:1) for $4 \mathrm{~h}$ to get 4 -acyl substituted methyl-1-cinnamoyl-1,4-diazepane-2-carboxylate derivatives. In the last step, 4-acyl substituted

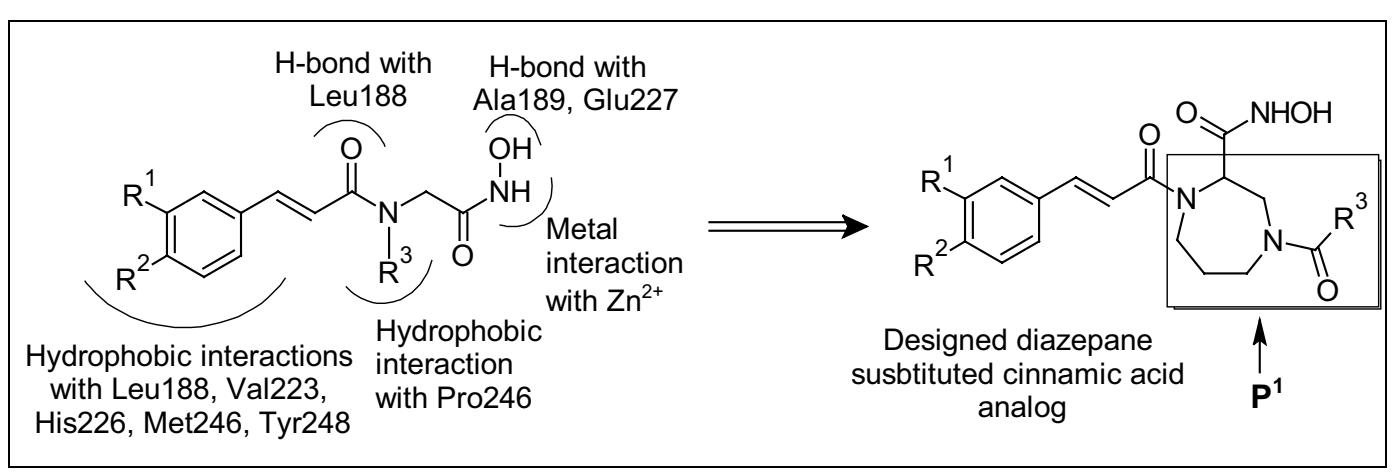

Fig. 1 General structure of the designed diazepine substituted cinnamic acid molecule 
methyl-1-cinnamoyl-1,4-diazepane-2-carboxylate derivatives were stirred with hydroxylamine (1:1), in methanol for $15 \mathrm{~min}$ to obtain the corresponding final products $[4,7,8,11,19]$. The reaction products were poured into crushed ice and precipitates which separated out were filtered, dried and recrystallized from ethanol. The synthesis was monitored by TLC on silica gel G Plates.

\section{4-Benzoyl-1-cinnamoyl-N-hydroxy-1,4-diazepane-2-carbox- amide (1)}

$\mathrm{Mp}\left({ }^{\circ} \mathrm{C}\right)$ 210; Yield-68.2\%; IR (KBr pellets, $\left.\mathrm{cm}^{-1}\right)$ : 1174 (C-O), $1288(-\mathrm{C}-\mathrm{N}), 1423(-\mathrm{C}-\mathrm{H}), 1529(\mathrm{C}=\mathrm{C})$, $1614(\mathrm{C}=\mathrm{N}), 1791(\mathrm{C}=\mathrm{O}), 2094(\mathrm{C} \equiv \mathrm{C}), 2324(\mathrm{C} \equiv \mathrm{N})$, $2677(-\mathrm{C}-\mathrm{H}=\mathrm{O}), 2742(-\mathrm{C}-\mathrm{H}=\mathrm{O}), 2937(\mathrm{C}-\mathrm{H}), 2976$ (=C-H), $3064(=\mathrm{C}-\mathrm{H}), 3431(\mathrm{~N}-\mathrm{H}), 3712(\mathrm{O}-\mathrm{H}) ;{ }^{1} \mathrm{H}$ NMR (DMSO- $\left.d_{6}, \delta \mathrm{ppm}\right): 2.010(\mathrm{~s}, 1 \mathrm{H}, \mathrm{OH}$ of $\mathrm{NHOH})$, 8.112 (s, 1H, NH of CONHOH), 3.213-5.161 (m, 9H, diazepane), 7.334-7.643 (m, $10 \mathrm{H}, \mathrm{CH}$ of $\left.\mathrm{C}_{6} \mathrm{H}_{5}\right), 7.337$ (s, 1H, CH of ethylene), 7.176 (s, $1 \mathrm{H}, \mathrm{CH}$ of ethylene); ${ }^{13} \mathrm{CNMR}$ (DMSO- $\left.d_{6}, \delta \mathrm{ppm}\right): 166.29\left(\mathrm{C}=\mathrm{O}\right.$ of $\left.\mathrm{COC}_{6} \mathrm{H}_{5}\right)$, $167.75(\mathrm{C}=\mathrm{O}$ of $\mathrm{CONHOH}), 167.49(\mathrm{C}=\mathrm{O}$ of amide), 144.54 (CH of ethylene), 136.15 (C of phenyl), 129.02 ( $\mathrm{CH}$ of phenyl), 126.96 ( $\mathrm{CH}$ of phenyl), 127.88 ( $\mathrm{CH}$ of phenyl), 130.23 ( $\mathrm{CH}$ of phenyl), 130.00 ( $\mathrm{CH}$ of phenyl), $134.57\left(\mathrm{C}\right.$ of $\left.\mathrm{COC}_{6} \mathrm{H}_{5}\right), 125.92\left(\mathrm{CH}\right.$ of $\left.\mathrm{COC}_{6} \mathrm{H}_{5}\right), 130.50$ $\left(\mathrm{CH}\right.$ of $\left.\mathrm{COC}_{6} \mathrm{H}_{5}\right), 129.72\left(\mathrm{CH}\right.$ of $\left.\mathrm{COC}_{6} \mathrm{H}_{5}\right), 128.66(\mathrm{CH}$ of $\left.\mathrm{COC}_{6} \mathrm{H}_{5}\right), 125.96\left(\mathrm{CH}\right.$ of $\left.\mathrm{COC}_{6} \mathrm{H}_{5}\right), 128.66(\mathrm{CH}$ of ethylene), $52.98\left(\mathrm{CH}\right.$, diazepane), $44.87\left(\mathrm{CH}_{2}\right.$, diazepane), 40.64 $\left(\mathrm{CH}_{2}\right.$, diazepane), $28.52\left(\mathrm{CH}_{2}\right.$, diazepane $), 40.01$ $\left(\mathrm{CH}_{2}\right.$, diazepane).

\section{4-Butyryl-1-cinnamoyl-N-hydroxy-1,4-diazepane-2-carbox- amide (2)}

Mp ( $\left.{ }^{\circ} \mathrm{C}\right)$ 121-123; Yield-68.9\%; IR ( $\mathrm{KBr}$ pellets, $\mathrm{cm}^{-1}$ ): $1172(\mathrm{C}-\mathrm{O}), 1249(-\mathrm{C}-\mathrm{N}), 1431(-\mathrm{C}-\mathrm{H}), 1581(\mathrm{C}=\mathrm{C})$, $1625(\mathrm{C}=\mathrm{N}), 1707(\mathrm{C}=\mathrm{O}), 1724(\mathrm{C}=\mathrm{O}), 2133(\mathrm{C} \equiv \mathrm{C})$, $2241(\mathrm{C} \equiv \mathrm{N}), 2692(-\mathrm{C}-\mathrm{H}=\mathrm{O}), 2877(\mathrm{C}-\mathrm{H}), 3049$ (=C$\mathrm{H}), 3068(=\mathrm{C}-\mathrm{H}), 3201(\equiv \mathrm{C}-\mathrm{H}), 3408(\mathrm{~N}-\mathrm{H}), 3712$ $(\mathrm{O}-\mathrm{H}) ;{ }^{1} \mathrm{H}$ NMR (DMSO- $\left.d_{6}, \delta \mathrm{ppm}\right): 1.961(\mathrm{~s}, 1 \mathrm{H}, \mathrm{OH}$ of $\mathrm{NHOH}), 8.104(\mathrm{~s}, 1 \mathrm{H}, \mathrm{NH}$ of $\mathrm{CONHOH}), 1.685-$ 5.261 (m, 9H, diazepane), 6.945-7.961 (m, 5H, $\mathrm{CH}$ of $\left.\mathrm{C}_{6} \mathrm{H}_{5}\right), 7.486(\mathrm{~s}, 1 \mathrm{H}, \mathrm{CH}$ of ethylene), $6.985(\mathrm{~s}, 1 \mathrm{H}, \mathrm{CH}$ of ethylene), $0.965\left(\mathrm{~m}, 3 \mathrm{H}, \mathrm{CH}_{3}\right.$ of $\left.\mathrm{COC}_{3} \mathrm{H}_{7}\right), 1.684(\mathrm{~m}$, $2 \mathrm{H}, \mathrm{CH}_{2}$ of $\left.\mathrm{COC}_{3} \mathrm{H}_{7}\right), 2.780\left(\mathrm{~m}, 2 \mathrm{H}, \mathrm{CH}_{2}\right.$ of $\left.\mathrm{COC}_{3} \mathrm{H}_{7}\right)$; ${ }^{13} \mathrm{CNMR}$ (DMSO- $\left.d_{6}, \delta \mathrm{ppm}\right): 167.70\left(\mathrm{C}=\mathrm{O}\right.$ of $\left.\mathrm{COC}_{3} \mathrm{H}_{7}\right)$, $167.46(\mathrm{C}=\mathrm{O}$ of $\mathrm{CONHOH}), 166.27(\mathrm{C}=\mathrm{O}$ of amide), 144.54 ( $\mathrm{CH}$ of ethylene), 136.15 (C of phenyl), 132.37 ( $\mathrm{CH}$ of phenyl), 131.23 ( $\mathrm{CH}$ of phenyl), 131.15 ( $\mathrm{CH}$ of phenyl), 130.50 ( $\mathrm{CH}$ of phenyl), 127.46 ( $\mathrm{CH}$ of phenyl), 128.66 ( $\mathrm{CH}$ of ethylene), $61.45(\mathrm{CH}$, diazepane), $52.98\left(\mathrm{CH}_{2}\right.$, diazepane), $44.87\left(\mathrm{CH}_{2}\right.$, diazepane $), 28.90$ $\left(\mathrm{CH}_{2}\right.$, diazepane $), 40.61\left(\mathrm{CH}_{2}\right.$, diazepane $), 39.39\left(\mathrm{CH}_{2}\right.$ of $\left.\mathrm{COC}_{3} \mathrm{H}_{7}\right), 28.52\left(\mathrm{CH}_{2}\right.$ of $\left.\mathrm{COC}_{3} \mathrm{H}_{7}\right), 17.66\left(\mathrm{CH}_{3}\right.$ of $\left.\mathrm{COC}_{3} \mathrm{H}_{7}\right) \cdot \mathrm{MS} \mathrm{ES}+(\mathrm{ToF}): \mathrm{m} / \mathrm{z} 360.4$.

\section{4-Acetyl-1-cinnamoyl-N-hydroxy-1,4-diazepane-2-carboxa- mide (3)}

$\mathrm{Mp}\left({ }^{\circ} \mathrm{C}\right)$ 164; Yield-67.8\%; IR (KBr pellets, $\left.\mathrm{cm}^{-1}\right): 1174$ (C-O), 1288 (-C-N), $1423(-\mathrm{C}-\mathrm{H}), 1529$ (C=C), 1614 $(\mathrm{C}=\mathrm{N}), 1791(\mathrm{C}=\mathrm{O}), 2094(\mathrm{C} \equiv \mathrm{C}), 2324(\mathrm{C} \equiv \mathrm{N}), 2677$ $(-\mathrm{C}-\mathrm{H}=\mathrm{O}), 2742(-\mathrm{C}-\mathrm{H}=\mathrm{O}), 2937(\mathrm{C}-\mathrm{H}), 2976$ (=C$\mathrm{H}), 3064(=\mathrm{C}-\mathrm{H}), 3431(\mathrm{~N}-\mathrm{H}), 3712(\mathrm{O}-\mathrm{H}) ;{ }^{1} \mathrm{H}$ NMR (DMSO- $\left.d_{6}, \delta \mathrm{ppm}\right): 2.012(\mathrm{~s}, 1 \mathrm{H}, \mathrm{OH}$ of $\mathrm{NHOH}), 8.114(\mathrm{~s}$, $1 \mathrm{H}, \mathrm{NH}$ of $\mathrm{CONHOH}), 1.712-5.161$ (m, 9H, diazepane), 6.562-7.534 (m, 5H, CH of $\left.\mathrm{C}_{6} \mathrm{H}_{5}\right), 7.486(\mathrm{~s}, 1 \mathrm{H}, \mathrm{CH}$ of ethylene), 6.985 (s, 1H, CH of ethylene), $2.732(\mathrm{~m}, 3 \mathrm{H}$, $\mathrm{CH}_{3}$ of $\left.\mathrm{COCH}_{3}\right) ;{ }^{13} \mathrm{CNMR}$ (DMSO- $\left.d_{6}, \delta \mathrm{ppm}\right): 172.52$ $\left(\mathrm{C}=\mathrm{O}\right.$ of $\left.\mathrm{COCH}_{3}\right), 171.12(\mathrm{C}=\mathrm{O}$ of $\mathrm{CONHOH}), 164.42$ ( $\mathrm{C}=\mathrm{O}$ of amide), 144.48 ( $\mathrm{CH}$ of ethylene), 138.59 ( $\mathrm{C}$ of phenyl), 133.77 ( $\mathrm{CH}$ of phenyl), 135.82 ( $\mathrm{CH}$ of phenyl), 134.82 ( $\mathrm{CH}$ of phenyl), 133.27 ( $\mathrm{CH}$ of phenyl), 133.06 ( $\mathrm{CH}$ of phenyl), 130.84 ( $\mathrm{CH}$ of ethylene), $35.46\left(\mathrm{CH}_{2}, \mathrm{C}_{6}\right.$ of diazepane), $45.98\left(\mathrm{CH}_{2}, \mathrm{C}_{7}\right.$ of diazepane $), 26.95\left(\mathrm{CH}_{3}\right.$ of $\mathrm{COCH}_{3}$ ); MS ES + (ToF): m/z 332.3.

\section{1-Cinnamoyl-N-hydroxy-4-propionyl-1,4-diazepane-2-car- boxamide (4)}

Mp ( $\left.{ }^{\circ} \mathrm{C}\right)$ 190; Yield-69.2\%; IR (KBr pellets, $\left.\mathrm{cm}^{-1}\right): 1172$ (C-O), $1286(-\mathrm{C}-\mathrm{N}), 1394(-\mathrm{C}-\mathrm{H}), 1435(-\mathrm{C}-\mathrm{H}), 1546$ $(\mathrm{C}=\mathrm{C}), 1629(\mathrm{C}=\mathrm{N}), 1707(\mathrm{C}=\mathrm{O}), 1714(\mathrm{C}=\mathrm{O}), 1737$ $(\mathrm{C}=\mathrm{O}), 2135(\mathrm{C} \equiv \mathrm{C}), 2239(\mathrm{C} \equiv \mathrm{N}), 2681(-\mathrm{C}-\mathrm{H}=\mathrm{O})$, $2744(-\mathrm{C}-\mathrm{H}=\mathrm{O}), 2935(\mathrm{C}-\mathrm{H}), 3421(\mathrm{~N}-\mathrm{H}), 3433(\mathrm{~N}-\mathrm{H})$, $3687(\mathrm{O}-\mathrm{H}), 3711(\mathrm{O}-\mathrm{H}) ;{ }^{1} \mathrm{H}$ NMR (DMSO-d 6 , $\delta$ ppm): 1.985 (s, $1 \mathrm{H}, \mathrm{OH}$ of $\mathrm{NHOH}), 8.015$ (s, 1H, NH of CON$\mathrm{HOH}), 2.712-5.161$ (m, 9H, diazepane), 7.355-7.523 (m, $5 \mathrm{H}, \mathrm{CH}$ of $\mathrm{C}_{6} \mathrm{H}_{5}$ ), 7.334 (s, $1 \mathrm{H}, \mathrm{CH}$ of ethylene), 6.981 (s, $1 \mathrm{H}, \mathrm{CH}$ of ethylene), $1.112\left(\mathrm{~m}, 3 \mathrm{H}, \mathrm{CH}_{3}\right.$ of $\left.\mathrm{COC}_{2} \mathrm{H}_{5}\right)$, $2.112\left(\mathrm{~m}, 2 \mathrm{H}, \mathrm{CH}_{2}\right.$ of $\left.\mathrm{COC}_{2} \mathrm{H}_{5}\right) ;{ }^{13} \mathrm{CNMR}$ (DMSO- $d_{6}, \delta$ ppm): $158.82\left(\mathrm{C}=\mathrm{O}\right.$ of $\left.\mathrm{COC}_{2} \mathrm{H}_{5}\right), 167.72(\mathrm{C}=\mathrm{O}$ of $\mathrm{CON}-$ $\mathrm{HOH}), 168.25$ ( $\mathrm{C}=\mathrm{O}$ of amide), 138.08 ( $\mathrm{CH}$ of ethylene), 134.81 (C of phenyl), 130.82 ( $\mathrm{CH}$ of phenyl), $124.53(\mathrm{CH}$ of phenyl), 122.53 ( $\mathrm{CH}$ of phenyl), 132.84 ( $\mathrm{CH}$ of phenyl), 130.79 ( $\mathrm{CH}$ of phenyl), $128.66(\mathrm{CH}$ of ethylene), 40.05 ( $\mathrm{CH}$, diazepane), $32.70\left(\mathrm{CH}_{2}\right.$, diazepane), 32.63 $\left(\mathrm{CH}_{2}\right.$, diazepane), 24.04 $\left(\mathrm{CH}_{2}\right.$, diazepane), $40.01\left(\mathrm{CH}_{2}\right.$, diazepane), $10.01\left(\mathrm{CH}_{2}\right.$ of $\left.\mathrm{COC}_{2} \mathrm{H}_{5}\right), 24.11\left(\mathrm{CH}_{3}\right.$ of $\left.\mathrm{COC}_{2} \mathrm{H}_{5}\right)$.

\section{4-Acetyl-N-hydroxy-1-(3-(3-hydroxyphenyl)acryloyl)-1,4-di- azepane-2-carboxamide (5)}

$\mathrm{Mp}\left({ }^{\circ} \mathrm{C}\right)$ 123; Yield-67.1\%; IR (KBr pellets, $\mathrm{cm}^{-1}$ ): 1217 (C-O), 1394 (-C-H), 1436 (-CH3), 1581 (-C-H), 1622 $(\mathrm{C}=\mathrm{N}), 1747(\mathrm{C}=\mathrm{O}), 1793(\mathrm{C}=\mathrm{O}), 1865(\mathrm{C}-\mathrm{H}), 2135$ $(\mathrm{C} \equiv \mathrm{C}), 2239(-\mathrm{C} \equiv \mathrm{N}), 2738$ (-CHO), 2758 (-CHO), 2945 
(C-H), 2966 (=C-H), $3190(\equiv \mathrm{C}-\mathrm{H}), 3446(\mathrm{~N}-\mathrm{H}), 3709$ $(\mathrm{O}-\mathrm{H}) ;{ }^{1} \mathrm{H}$ NMR (DMSO- $\left.d_{6}, \delta \mathrm{ppm}\right): 2.010$ (s, $1 \mathrm{H}, \mathrm{OH}$ of $\mathrm{NHOH}), 8.112$ (s, $1 \mathrm{H}, \mathrm{NH}$ of $\mathrm{CONHOH}), 2.780-5.161$ (m, 9H, diazepane), 7.334-7.535 (m, 4H, CH, aromatic), 7.334 (s, $1 \mathrm{H}, \mathrm{CH}$ of ethylene), $7.176(\mathrm{~s}, 1 \mathrm{H}, \mathrm{CH}$ of ethylene), $1.892\left(\mathrm{~m}, 3 \mathrm{H}, \mathrm{CH}_{3}\right.$ of $\left.\mathrm{COCH}_{3}\right), 5.321$ (s, H, aromatic $\mathrm{OH}) ;{ }^{13} \mathrm{CNMR}$ (DMSO- $\left.d_{6}, \delta \mathrm{ppm}\right): 158.58(\mathrm{C}=\mathrm{O}$ of $\left.\mathrm{COCH}_{3}\right), 167.72(\mathrm{C}=\mathrm{O}$ of $\mathrm{CONHOH}), 168.25(\mathrm{C}=\mathrm{O}$ of amide), 138.08 ( $\mathrm{CH}$ of ethylene), 134.81 (C of phenyl), 132.34 (CH of phenyl), 130.82 (C of phenyl), $124.15(\mathrm{CH}$ of phenyl), 122.34 ( $\mathrm{CH}$ of phenyl), $122.53(\mathrm{CH}$ of phenyl), 130.29 ( $\mathrm{CH}$ of ethylene), $40.06(\mathrm{CH}$, diazepane), $39.68\left(\mathrm{CH}_{2}\right.$, diazepane), $39.22\left(\mathrm{CH}_{2}\right.$, diazepane $), 24.04$ $\left(\mathrm{CH}_{2}\right.$, diazepane $), 40.02\left(\mathrm{CH}_{2}\right.$, diazepane $), 24.11\left(\mathrm{CH}_{3}\right.$ of $\mathrm{COCH}_{3}$ ).

\section{N-Hydroxy-1-(3-(3-hydroxyphenyl)acryloyl)-4-propio- nyl-1,4-diazepane-2-carboxamide (6)}

Mp ( $\left.{ }^{\circ} \mathrm{C}\right)$ 125-127; Yield-69.4\%; IR ( $\mathrm{KBr}$ pellets, $\left.\mathrm{cm}^{-1}\right): 1170$ (C-O), $1396(\mathrm{C}-\mathrm{H}), 1438\left(\mathrm{CH}_{3}\right), 1627$ $(\mathrm{C}=\mathrm{N}), 2133(\mathrm{C} \equiv \mathrm{C}), 2239(\mathrm{C} \equiv \mathrm{N}), 2947(\mathrm{C}-\mathrm{H}), 3057$ $(=\mathrm{C}-\mathrm{H}), 3136(\equiv \mathrm{C}-\mathrm{H}), 3452(\mathrm{~N}-\mathrm{H}), 3765(\mathrm{O}-\mathrm{H}) ;{ }^{1} \mathrm{H}$ NMR (DMSO- $\left.d_{6}, \delta \mathrm{ppm}\right): 1.984(\mathrm{~s}, 1 \mathrm{H}, \mathrm{OH}$ of $\mathrm{NHOH})$, $8.015(\mathrm{~s}, 1 \mathrm{H}, \mathrm{NH}$ of $\mathrm{CONHOH}), 5.386(\mathrm{H}, \mathrm{OH}$, aromatic), 3.134-5.016 ( $\mathrm{m}, 9 \mathrm{H}$, diazepane), 6.945-7.535 (m, $4 \mathrm{H}, \mathrm{CH}$ of $\mathrm{C}_{6} \mathrm{H}_{5}$ ), 7.334 (s, $1 \mathrm{H}, \mathrm{CH}$ of ethylene), 7.176 (s, $1 \mathrm{H}, \mathrm{CH}$ of ethylene), $1.235\left(\mathrm{~m}, 2 \mathrm{H}, \mathrm{CH}_{2}\right.$ of $\left.\mathrm{COCH}_{2} \mathrm{CH}_{3}\right)$, $2.235\left(\mathrm{~m}, 3 \mathrm{H}, \mathrm{CH}_{3}\right.$ of $\left.\mathrm{COCH}_{2} \mathrm{CH}_{3}\right) ;{ }^{13} \mathrm{CNMR}$ (DMSO$d_{6}, \delta$ ppm): $168.25\left(\mathrm{C}=\mathrm{O}\right.$ of $\left.\mathrm{COCH}_{2} \mathrm{CH}_{3}\right), 167.72(\mathrm{C}=\mathrm{O}$ of $\mathrm{CONHOH}), 158.58(\mathrm{C}=\mathrm{O}$ of amide), $138.08(\mathrm{CH}$ of ethylene), 134.81 ( $\mathrm{C}$ of phenyl), $122.26(\mathrm{CH}$ of phenyl), 130.82 (CH of phenyl), 115.22 ( $\mathrm{CH}$ of phenyl), 158.49 (C of phenyl), 118.28 ( $\mathrm{CH}$ of phenyl), 122.34 ( $\mathrm{CH}$ of ethylene), $24.11\left(\mathrm{CH}_{2}\right.$, diazepane $), 40.02\left(\mathrm{CH}_{2}\right.$, diazepane), $32.63\left(\mathrm{CH}_{2}\right.$ of $\left.\mathrm{COCH}_{2} \mathrm{CH}_{3}\right), 24.04\left(\mathrm{CH}_{3}\right.$ of $\left.\mathrm{COCH}_{2} \mathrm{CH}_{3}\right)$; MS ES + (ToF): m/z 362.3.

\section{4-Benzoyl-N-hydroxy-1-(3-(3-hydroxyphenyl)acryloyl)- 1,4-diazepane-2-carboxamide (7)}

Mp ( $\left.{ }^{\circ} \mathrm{C}\right)$ 230-231; Yield-66.5\%; IR (KBr pellets, $\mathrm{cm}^{-1}$ ): 1172 (C-O), 1288 (C-N), 1396 (C-H), 1423 (CH3), 1581 $(\mathrm{C}=\mathrm{C}), 1676(\mathrm{C}=\mathrm{N}), 1793(\mathrm{C}=\mathrm{O}), 2090(\mathrm{C} \equiv \mathrm{C}), 2241$ $(\mathrm{C} \equiv \mathrm{N}), 2843(\mathrm{C}-\mathrm{H}), 2910(=\mathrm{C}-\mathrm{H}), 3030(=\mathrm{C}-\mathrm{H}), 3155$ $(\equiv \mathrm{C}-\mathrm{H}), 3423(\mathrm{~N}-\mathrm{H}), 3770(\mathrm{O}-\mathrm{H}) ;{ }^{1} \mathrm{H}$ NMR (DMSO-d ${ }_{6}$, $\delta \mathrm{ppm}): 2.016(\mathrm{~s}, 1 \mathrm{H}, \mathrm{OH}$ of $\mathrm{NHOH}), 8.121(\mathrm{~s}, 1 \mathrm{H}, \mathrm{NH}$ of $\mathrm{CONHOH}), 5.361(\mathrm{H}, \mathrm{OH}$, aromatic), 1.891-5.012 (m, 9H, diazepane), 6.945-7.535 (m, 9H, CH, aromatic), 7.334 (s, $1 \mathrm{H}, \mathrm{CH}$ of ethylene), 6.985 (s, $1 \mathrm{H}, \mathrm{CH}$ of ethylene); ${ }^{13} \mathrm{CNMR}$ (DMSO- $\left.d_{6}, \delta \mathrm{ppm}\right): 167.42(\mathrm{C}=\mathrm{O}$ of $\left.\mathrm{COC}_{6} \mathrm{H}_{5}\right), 167.83(\mathrm{C}=\mathrm{O}$ of $\mathrm{CONHOH}), 166.36(\mathrm{C}=\mathrm{O}$ of amide), 141.38 ( $\mathrm{CH}$ of ethylene), 134.36 ( $\mathrm{C}$ of phenyl), 124.09 ( $\mathrm{CH}$ of phenyl), 148.22 (C of phenyl), $122.12(\mathrm{CH}$ of phenyl), 130.79 ( $\mathrm{CH}$ of phenyl), 122.12 ( $\mathrm{CH}$ of phenyl),
$134.81\left(\mathrm{C}\right.$ of $\left.\mathrm{COC}_{6} \mathrm{H}_{5}\right), 124.15\left(\mathrm{CH}\right.$ of $\left.\mathrm{COC}_{6} \mathrm{H}_{5}\right), 130.82$ $\left(\mathrm{CH}\right.$ of $\left.\mathrm{COC}_{6} \mathrm{H}_{5}\right), 130.82\left(\mathrm{CH}\right.$ of $\left.\mathrm{COC}_{6} \mathrm{H}_{5}\right), 122.53(\mathrm{CH}$ of $\left.\mathrm{COC}_{6} \mathrm{H}_{5}\right), 122.34\left(\mathrm{CH}\right.$ of $\left.\mathrm{COC}_{6} \mathrm{H}_{5}\right), 130.79(\mathrm{CH}$ of ethylene), 40.08 ( $\mathrm{CH}$, diazepane), $39.52\left(\mathrm{CH}_{2}\right.$, diazepane), $39.02\left(\mathrm{CH}_{2}\right.$, diazepane $), 40.01\left(\mathrm{CH}_{2}\right.$, diazepane); $\mathrm{MS}$ $\mathrm{ES}+(\mathrm{ToF}): \mathrm{m} / \mathrm{z} 410.4$.

\section{4-Butyryl-N-hydroxy-1-(3-(3-hydroxyphenyl)acryloyl)-1,4-di- azepane-2-carboxamide (8)}

$\mathrm{Mp}\left({ }^{\circ} \mathrm{C}\right)$ 255-255.5; Yield-67.3\%; IR (KBr pellets, $\left.\mathrm{cm}^{-1}\right)$ : $1170(\mathrm{C}-\mathrm{O}), 1278(\mathrm{C}-\mathrm{N}), 1400(\mathrm{C}-\mathrm{H}), 1581(\mathrm{C}=\mathrm{C}), 1622$ $(\mathrm{C}=\mathrm{N}), 1737(\mathrm{C}=\mathrm{O}), 2086(\mathrm{C} \equiv \mathrm{C}), 2241(\mathrm{C} \equiv \mathrm{N}), 2935$ (C-H), 2978 (=C-H), $3047(=\mathrm{C}-\mathrm{H}), 3182$ (三C-H), $3427(\mathrm{~N}-\mathrm{H}), 3770(\mathrm{O}-\mathrm{H}) ;{ }^{1} \mathrm{H}$ NMR (DMSO- $\left.d_{6}, \delta \mathrm{ppm}\right)$ : $2.002(\mathrm{~s}, 1 \mathrm{H}, \mathrm{OH}$ of $\mathrm{NHOH}), 8.112(\mathrm{~s}, 1 \mathrm{H}, \mathrm{NH}$ of CON$\mathrm{HOH}), 4.984(\mathrm{H}, \mathrm{OH}$, aromatic), 2.732-5.161 (m, 9H, diazepane), 7.235-7.523 (m, 4H, $\mathrm{CH}$ of $\left.\mathrm{C}_{6} \mathrm{H}_{5}\right), 7.334$ (s, $1 \mathrm{H}, \mathrm{CH}$ of ethylene), $7.176(\mathrm{~s}, 1 \mathrm{H}, \mathrm{CH}$ of ethylene), $2.712\left(\mathrm{~m}, 2 \mathrm{H}, \mathrm{CH}_{2}\right.$ of $\left.\mathrm{COC}_{3} \mathrm{H}_{7}\right), 1.011\left(\mathrm{~m}, 3 \mathrm{H}, \mathrm{CH}_{3}\right.$ of $\left.\mathrm{COC}_{3} \mathrm{H}_{7}\right) ;{ }^{13} \mathrm{CNMR}$ (DMSO- $\left.d_{6}, \delta \mathrm{ppm}\right): 167.72(\mathrm{C}=\mathrm{O}$ of $\left.\mathrm{COC}_{3} \mathrm{H}_{7}\right), 168.25(\mathrm{C}=\mathrm{O}$ of $\mathrm{CONHOH}), 158.58(\mathrm{C}=\mathrm{O}$ of amide), 138.08 ( $\mathrm{CH}$ of ethylene), 134.81 (C of phenyl), 116.71 ( $\mathrm{CH}$ of phenyl), 158.49 ( $\mathrm{C}$ of phenyl), $115.22(\mathrm{CH}$ of phenyl), 130.29 ( $\mathrm{C}$ of phenyl), 118.28 ( $\mathrm{CH}$ of phenyl), 130.79 ( $\mathrm{CH}$ of ethylene), 40.06 ( $\mathrm{CH}$ of diazepane), 24.04 $\left(\mathrm{CH}_{2}\right.$ of diazepane), $32.63\left(\mathrm{CH}_{2}\right.$ of diazepane $), 32.70$ $\left(\mathrm{CH}_{2}\right.$ of $\left.\mathrm{COC}_{3} \mathrm{H}_{7}\right), 24.11\left(\mathrm{CH}_{2}\right.$ of $\left.\mathrm{COC}_{3} \mathrm{H}_{7}\right), 18.28\left(\mathrm{CH}_{3}\right.$ of $\mathrm{COC}_{3} \mathrm{H}_{7}$ ).

\section{4-Acetyl-N-hydroxy-1-(3-(4-hydroxyphenyl)acryloyl)-1,4-di- azepane-2-carboxamide (9)}

Mp ( $\left.{ }^{\circ} \mathrm{C}\right)$ 124-125; Yield-68.1\%; IR ( $\mathrm{KBr}$ pellets, $\left.\mathrm{cm}^{-1}\right)$ : 1172 (C-O), $1276(-\mathrm{C}-\mathrm{N}), 1433(-\mathrm{C}-\mathrm{H}), 1581(\mathrm{C}=\mathrm{C})$, $1627(\mathrm{C}=\mathrm{N}), 1732(\mathrm{C}=\mathrm{O}), 2135(\mathrm{C} \equiv \mathrm{C}), 2239(\mathrm{C} \equiv \mathrm{N})$, $2677(-\mathrm{C}-\mathrm{H}=\mathrm{O}), 2742(-\mathrm{C}-\mathrm{H}=\mathrm{O}), 2935(\mathrm{C}-\mathrm{H}), 2970$ (=C-H), 3059 (=C-H), 3167 ( $=\mathrm{C}-\mathrm{H}), 3414(\mathrm{~N}-\mathrm{H}), 3504$ $(\mathrm{O}-\mathrm{H}), 3753(\mathrm{O}-\mathrm{H}) ;{ }^{1} \mathrm{H}$ NMR (DMSO-d $\left.{ }_{6}, \delta \mathrm{ppm}\right): 2.010$ (s, $1 \mathrm{H}, \mathrm{OH}$ of $\mathrm{NHOH}), 8.110(\mathrm{~s}, 1 \mathrm{H}, \mathrm{NH}$ of $\mathrm{CONHOH})$, 5.462 (s, H, aromatic $\mathrm{OH}), 2.761-5.161$ (m, 9H, diazepane), 6.562-7.534 (m, 4H, CH of $\left.\mathrm{C}_{6} \mathrm{H}_{5}\right), 7.334(\mathrm{~s}, 1 \mathrm{H}, \mathrm{CH}$ of ethylene), 7.217 (s, $1 \mathrm{H}, \mathrm{CH}$ of ethylene), $2.712\left(\mathrm{~m}, 3 \mathrm{H}, \mathrm{CH}_{3}\right.$ of $\left.\mathrm{COCH}_{3}\right) ;{ }^{13} \mathrm{CNMR}$ (DMSO- $\left.d_{6}, \delta \mathrm{ppm}\right): 168.25(\mathrm{C}=\mathrm{O}$ of $\left.\mathrm{COCH}_{3}\right), 167.72(\mathrm{C}=\mathrm{O}$ of $\mathrm{CONHOH}), 158.58(\mathrm{C}=\mathrm{O}$ of amide), 138.08 ( $\mathrm{CH}$ of ethylene), 124.15 ( $\mathrm{C}$ of phenyl), 130.79 ( $\mathrm{CH}$ of phenyl), 116.71 ( $\mathrm{CH}$ of phenyl), $158.49(\mathrm{C}$ of phenyl), 115.22 ( $\mathrm{CH}$ of phenyl), $130.82(\mathrm{CH}$ of phenyl), 122.53 ( $\mathrm{CH}$ of ethylene), $24.11\left(\mathrm{CH}_{2}\right.$, diazepane), 40.02 $\left(\mathrm{CH}_{2}\right.$, diazepane), $26.95\left(\mathrm{CH}_{3}\right.$ of $\left.\mathrm{COCH}_{3}\right)$.

\section{N-hydroxy-1-(3-(4-hydroxyphenyl)acryloyl)-4-propio- nyl-1,4-diazepane-2-carboxamide (10)}

$\mathrm{Mp}\left({ }^{\circ} \mathrm{C}\right)$ 135; Yield-69.1\%; IR (KBr pellets, $\mathrm{cm}^{-1}$ ): 1172 (C-O), $1400(-\mathrm{C}-\mathrm{H}), 1581(\mathrm{C}=\mathrm{C}), 1622(\mathrm{C}=\mathrm{N}), 1732$ 
$(\mathrm{C}=\mathrm{O}), 2140(\mathrm{C} \equiv \mathrm{C}), 2245(\mathrm{C} \equiv \mathrm{N}), 2681(-\mathrm{C}-\mathrm{H}=\mathrm{O})$, $2735(-\mathrm{C}-\mathrm{H}=\mathrm{O}), 2937(\mathrm{C}-\mathrm{H}), 2953(\mathrm{C}-\mathrm{H}), 2999$ (=C$\mathrm{H}), 3043(=\mathrm{C}-\mathrm{H}), 3161(\equiv \mathrm{C}-\mathrm{H}), 3400(\mathrm{~N}-\mathrm{H}), 3429$ $(\mathrm{N}-\mathrm{H}), 3522(\mathrm{O}-\mathrm{H}), 3770(\mathrm{O}-\mathrm{H}) ;{ }^{1} \mathrm{H}$ NMR (DMSO-d ${ }_{6}$, $\delta \mathrm{ppm}): 2.010$ (s, $1 \mathrm{H}, \mathrm{OH}$ of $\mathrm{NHOH}), 8.110(\mathrm{~s}, 1 \mathrm{H}, \mathrm{NH}$ of $\mathrm{CONHOH}$ ), 5.462 (s, H, OH, aromatic), 2.761-5.161 (m, 9H, diazepane), 6.562-7.534 (m, 4H, $\mathrm{CH}$ of $\left.\mathrm{C}_{6} \mathrm{H}_{5}\right)$, 7.334 (s, 1H, CH of ethylene), 7.217 (s, 1H, CH of ethylene), $2.712\left(\mathrm{~m}, 2 \mathrm{H}, \mathrm{CH}_{2}\right.$ of $\left.\mathrm{COC}_{2} \mathrm{H}_{5}\right), 2.712\left(\mathrm{~m}, 3 \mathrm{H}, \mathrm{CH}_{3}\right.$ of $\left.\mathrm{COC}_{2} \mathrm{H}_{5}\right) ;{ }^{13} \mathrm{CNMR}$ (DMSO- $\left.d_{6}, \delta \mathrm{ppm}\right): 168.25(\mathrm{C}=\mathrm{O}$ of $\left.\mathrm{COC}_{2} \mathrm{H}_{5}\right), 167.72(\mathrm{C}=\mathrm{O}$ of $\mathrm{CONHOH}), 158.58(\mathrm{C}=\mathrm{O}$ of amide), 138.08 ( $\mathrm{CH}$ of ethylene), 124.15 (C of phenyl), 130.79 ( $\mathrm{CH}$ of phenyl), 118.71 ( $\mathrm{CH}$ of phenyl), 158.49 (C of phenyl), 115.22 ( $\mathrm{CH}$ of phenyl), 130.79 ( $\mathrm{CH}$ of phenyl), 122.53 ( $\mathrm{CH}$ of ethylene), $40.04(\mathrm{CH}$, diazepane), $39.42\left(\mathrm{CH}_{2}\right.$, diazepane), $32.70\left(\mathrm{CH}_{2}\right.$, diazepane $), 24.04$ $\left(\mathrm{CH}_{2}\right.$, diazepane), $32.60\left(\mathrm{CH}_{2}\right.$, diazepane $), 24.11\left(\mathrm{CH}_{2}\right.$ of $\mathrm{COC}_{2} \mathrm{H}_{5}$ ).

4-Benzoyl-N-hydroxy-1-(3-(4-hydroxyphenyl)acryloyl)-1,4-diazepane-2-carboxamide (11)

Mp ( $\left.{ }^{\circ} \mathrm{C}\right)$ 232-233; Yield-68.1\%; IR ( $\mathrm{KBr}$ pellets, $\left.\mathrm{cm}^{-1}\right): 1174$ (C-O), $1288(\mathrm{C}-\mathrm{N}), 1425(\mathrm{C}-\mathrm{H}), 1581$ $(\mathrm{C}=\mathrm{C}), 1616(\mathrm{C}=\mathrm{N}), 1629(\mathrm{C}=\mathrm{N}), 1699(\mathrm{C}=\mathrm{O}), 1791$ (C=O), $1928(-\mathrm{C}-\mathrm{H}), 2129(\mathrm{C} \equiv \mathrm{C}), 2241(\mathrm{C} \equiv \mathrm{N}), 2735$ $(-\mathrm{C}=\mathrm{O}-\mathrm{OH}), 2958(\mathrm{C}-\mathrm{H}), 2987(\mathrm{C}-\mathrm{H}), 3018(=\mathrm{C}-\mathrm{H})$, $3062(=\mathrm{C}-\mathrm{H}), \quad 3176.76(\equiv \mathrm{C}-\mathrm{H}), 3456(\mathrm{~N}-\mathrm{H}), 3469$ $(\mathrm{N}-\mathrm{H}), 3755(\mathrm{O}-\mathrm{H}), 3770(\mathrm{O}-\mathrm{H}) ;{ }^{1} \mathrm{H}$ NMR (DMSO-d ${ }_{6}$, $\delta \mathrm{ppm}): 2.013$ (s, $1 \mathrm{H}, \mathrm{OH}$ of $\mathrm{NHOH}), 8.104(\mathrm{~s}, 1 \mathrm{H}, \mathrm{NH}$ of $\mathrm{CONHOH}), 4.985$ (s, H, OH, aromatic), 1.705-5.215 (m, 9H, diazepane), 6.945-7.643 (m, 9H, CH, aromatic), 7.334 (s, $1 \mathrm{H}, \mathrm{CH}$ of ethylene), 7.176 (s, $1 \mathrm{H}, \mathrm{CH}$ of ethylene); ${ }^{13} \mathrm{CNMR}$ (DMSO- $\left.d_{6}, \delta \mathrm{ppm}\right): 168.25$ (C=O of $\left.\mathrm{COC}_{6} \mathrm{H}_{5}\right), 167.72(\mathrm{C}=\mathrm{O}$ of $\mathrm{CONHOH}), 158.58(\mathrm{C}=\mathrm{O}$ of amide), 138.08 ( $\mathrm{CH}$ of ethylene), 124.15 (C of phenyl), 130.79 (CH of phenyl), 116.71 ( $\mathrm{CH}$ of phenyl), 158.49 (C of phenyl), 115.22 ( $\mathrm{CH}$ of phenyl), 130.79 ( $\mathrm{CH}$ of phenyl), $134.81\left(\mathrm{C}\right.$ of $\left.\mathrm{COC}_{6} \mathrm{H}_{5}\right), 124.34\left(\mathrm{CH}\right.$ of $\left.\mathrm{COC}_{6} \mathrm{H}_{5}\right), 130.82$ $\left(\mathrm{CH}\right.$ of $\left.\mathrm{COC}_{6} \mathrm{H}_{5}\right), 132.34\left(\mathrm{CH}\right.$ of $\left.\mathrm{COC}_{6} \mathrm{H}_{5}\right), 130.82(\mathrm{CH}$ of $\left.\mathrm{COC}_{6} \mathrm{H}_{5}\right), 122.34\left(\mathrm{CH}\right.$ of $\left.\mathrm{COC}_{6} \mathrm{H}_{5}\right), 124.15$ ( $\mathrm{CH}$ of ethylene), 40.04 ( $\mathrm{CH}$, diazepane), $39.82\left(\mathrm{CH}_{2}\right.$, diazepane).

\section{4-Butyryl-N-hydroxy-1-(3-(4-hydroxyphenyl)acryloyl)-1,4-di- azepane-2-carboxamide (12)}

Mp $\left({ }^{\circ} \mathrm{C}\right)$ 251-252; Yield-67.8\%; IR (KBr pellets, $\mathrm{cm}^{-1}$ ): 1170 (C-O), $1325(\mathrm{C}-\mathrm{N}), 1431(\mathrm{C}-\mathrm{H}), 1581(\mathrm{C}=\mathrm{C}), 1622$ $(\mathrm{C}=\mathrm{N}), 1722(\mathrm{C}=\mathrm{O}), 1732(\mathrm{C}=\mathrm{O}), 2102(\mathrm{C}=\mathrm{O}), 2135$ $(\mathrm{C} \equiv \mathrm{C}), 2243(\mathrm{C} \equiv \mathrm{N}), 2742(-\mathrm{C}=\mathrm{O}-\mathrm{OH}), 2939(\mathrm{C}-\mathrm{H})$, $2974(=\mathrm{C}-\mathrm{H}), 3390(\equiv \mathrm{C}-\mathrm{H}), 3419(\mathrm{~N}-\mathrm{H}), 3444(\mathrm{~N}-\mathrm{H})$, $3743(\mathrm{O}-\mathrm{H}) ;{ }^{1} \mathrm{H}$ NMR (DMSO- $\left.d_{6}, \delta \mathrm{ppm}\right): 2.010(\mathrm{~s}, 1 \mathrm{H}$, $\mathrm{OH}$ of $\mathrm{NHOH}), 8.110$ (s, 1H, $\mathrm{NH}$ of $\mathrm{CONHOH}$ ), 4.910 (s, H, OH, aromatic), 1.171-5.161 (m, 9H, diazepane), 6.562-7.523 (m, 4H, CH, aromatic), 7.334 (s, 1H, CH of ethylene), $6.981(\mathrm{~s}, 1 \mathrm{H}, \mathrm{CH}$ of ethylene), $2.712(\mathrm{~m}$, $2 \mathrm{H}, \mathrm{CH}_{2}$ of $\left.\mathrm{COC}_{3} \mathrm{H}_{7}\right), 1.167\left(\mathrm{~m}, 3 \mathrm{H}, \mathrm{CH}_{3}\right.$ of $\left.\mathrm{COC}_{3} \mathrm{H}_{7}\right)$; ${ }^{13} \mathrm{CNMR}$ (DMSO- $\left.d_{6}, \delta \mathrm{ppm}\right): 168.25\left(\mathrm{C}=\mathrm{O}\right.$ of $\left.\mathrm{COC}_{3} \mathrm{H}_{7}\right)$, $167.72(\mathrm{C}=\mathrm{O}$ of $\mathrm{CONHOH}), 158.58(\mathrm{C}=\mathrm{O}$ of amide), 138.08 ( $\mathrm{CH}$ of ethylene), 124.15 (C of phenyl), 130.79 ( $\mathrm{CH}$ of phenyl), 118.71 ( $\mathrm{CH}$ of phenyl), 158.49 (C of phenyl), 115.22 ( $\mathrm{CH}$ of phenyl), 130.79 ( $\mathrm{CH}$ of phenyl), 122.53 ( $\mathrm{CH}$ of ethylene), 40.04 ( $\mathrm{CH}$, diazepane), 32.70 $\left(\mathrm{CH}_{2}\right.$, diazepane), $24.04\left(\mathrm{CH}_{2}\right.$, diazepane $), 32.63\left(\mathrm{CH}_{2}\right.$ of $\left.\mathrm{COC}_{3} \mathrm{H}_{7}\right), 24.11\left(\mathrm{CH}_{2}\right.$ of $\left.\mathrm{COC}_{3} \mathrm{H}_{7}\right), 18.28\left(\mathrm{CH}_{3}\right.$ of $\mathrm{COC}_{3} \mathrm{H}_{7}$ ).

\section{4-Acetyl-N-hydroxy-1-(3-(3,4-dihydroxyphenyl) acryloyl)-1,4-diazepane-2-carboxamide (13)}

$\mathrm{Mp}\left({ }^{\circ} \mathrm{C}\right)$ 180; Yield-68.1\%; IR (KBr pellets, $\left.\mathrm{cm}^{-1}\right): 1172$ (C-O), $1263(\mathrm{C}-\mathrm{N}), 1440(\mathrm{C}-\mathrm{H}), 1581(\mathrm{C}=\mathrm{C}), 1622$ $(\mathrm{C}=\mathrm{N}), 1793(\mathrm{C}=\mathrm{O}), 2129(\mathrm{C} \equiv \mathrm{C}), 2241(\mathrm{C} \equiv \mathrm{N}), 2677$ $(-\mathrm{C}=\mathrm{O}-\mathrm{OH}), 2935(\mathrm{C}-\mathrm{H}), 2976(\mathrm{C}-\mathrm{H}), 3057(=\mathrm{C}-\mathrm{H})$, $3101(=\mathrm{C}-\mathrm{H}), 3149(\equiv \mathrm{C}-\mathrm{H}), 3161(\equiv \mathrm{C}-\mathrm{H}), 3462(\mathrm{~N}-\mathrm{H})$, $3481(\mathrm{~N}-\mathrm{H}), 3755(\mathrm{O}-\mathrm{H}) ;{ }^{1} \mathrm{H}$ NMR (DMSO- $\left.d_{6}, \delta \mathrm{ppm}\right)$ : 2.010 (s, 1H, OH of $\mathrm{NHOH}), 8.091$ (s, 1H, NH of CON$\mathrm{HOH}), 4.918$ (d, 2H, aromatic $\mathrm{OH}), 2.873-5.161(\mathrm{~m}, 9 \mathrm{H}$, diazepane), 6.945-7.951 (m, 3H, $\mathrm{CH}$ of $\left.\mathrm{C}_{6} \mathrm{H}_{5}\right), 7.446$ (s, 1H, CH of ethylene), 6.985 (s, 1H, CH of ethylene), $2.780\left(\mathrm{~m}, 3 \mathrm{H}, \mathrm{CH}_{3}\right.$ of $\left.\mathrm{COCH}_{3}\right) ;{ }^{13} \mathrm{CNMR}$ (DMSO- $d_{6}, \delta$ ppm): $168.25\left(\mathrm{C}=\mathrm{O}\right.$ of $\left.\mathrm{COCH}_{3}\right), 167.72(\mathrm{C}=\mathrm{O}$ of $\mathrm{CON}-$ $\mathrm{HOH}), 158.58$ ( $\mathrm{C}=\mathrm{O}$ of amide), 138.08 ( $\mathrm{CH}$ of ethylene), 130.79 (C of phenyl), 116.71 ( $\mathrm{CH}$ of phenyl), 158.49 (C of phenyl), 158.58 ( $\mathrm{C}$ of phenyl), 118.28 ( $\mathrm{CH}$ of phenyl), 122.53 ( $\mathrm{CH}$ of phenyl), 124.15 ( $\mathrm{CH}$ of ethylene), 24.11 $\left(\mathrm{CH}_{2}\right.$, diazepane), $40.08(\mathrm{CH}$, diazepane $), 26.95\left(\mathrm{CH}_{3}\right.$ of $\mathrm{COCH}_{3}$ ).

\section{N-hydroxy-1-(3-(3,4-dihydroxyphenyl)acryloyl)-4-propio- nyl-1,4-diazepane-2-carboxamide (14)}

Mp $\left({ }^{\circ} \mathrm{C}\right)$ 200; Yield-69.2\%; IR (KBr pellets, $\left.\mathrm{cm}^{-1}\right): 1170$ (C-O), $1263(\mathrm{C}-\mathrm{N}), 1431(\mathrm{C}-\mathrm{H}), 1581(\mathrm{C}=\mathrm{C}), 1645$ $(\mathrm{C}=\mathrm{N}), 1720(\mathrm{C}=\mathrm{O}), 2113(\mathrm{C} \equiv \mathrm{C}), 2306(\mathrm{C} \equiv \mathrm{N}), 2692(-$ $\mathrm{C}=\mathrm{O}-\mathrm{OH}), 2893(\mathrm{C}-\mathrm{H}), 3022(=\mathrm{C}-\mathrm{H}), 3167(\equiv \mathrm{C}-\mathrm{H})$, $3265(\equiv \mathrm{C}-\mathrm{H}), 3433(\mathrm{~N}-\mathrm{H}), 3471(\mathrm{~N}-\mathrm{H}), 3520(\mathrm{O}-\mathrm{H})$, $3709(\mathrm{O}-\mathrm{H}) ;{ }^{1} \mathrm{H}$ NMR (DMSO- $\left.d_{6}, \delta \mathrm{ppm}\right): 2.010(\mathrm{~s}, 1 \mathrm{H}$, $\mathrm{OH}$ of $\mathrm{NHOH}$ ), 8.110 (s, 1H, NH of $\mathrm{CONHOH}), 4.910$ (d, 2H, aromatic $\mathrm{OH}), 2.765-5.161$ ( $\mathrm{m}, 9 \mathrm{H}$, diazepane), 6.562-7.523 $\left(\mathrm{m}, 3 \mathrm{H}, \mathrm{CH}\right.$ of $\left.\mathrm{C}_{6} \mathrm{H}_{5}\right), 7.334(\mathrm{~s}, 1 \mathrm{H}, \mathrm{CH}$ of ethylene), $6.981(\mathrm{~s}, 1 \mathrm{H}, \mathrm{CH}$ of ethylene), $2.712(\mathrm{~m}$, $2 \mathrm{H}, \mathrm{CH}_{2}$ of $\left.\mathrm{COC}_{2} \mathrm{H}_{5}\right), 1.171\left(\mathrm{~m}, 3 \mathrm{H}, \mathrm{CH}_{3}\right.$ of $\left.\mathrm{COC}_{2} \mathrm{H}_{5}\right)$; ${ }^{13} \mathrm{CNMR}$ (DMSO- $\left.d_{6}, \delta \mathrm{ppm}\right): 168.25\left(\mathrm{C}=\mathrm{O}\right.$ of $\left.\mathrm{COC}_{2} \mathrm{H}_{5}\right)$, $167.72(\mathrm{C}=\mathrm{O}$ of $\mathrm{CONHOH}), 158.58(\mathrm{C}=\mathrm{O}$ of amide), 138.08 ( $\mathrm{CH}$ of ethylene), 130.82 (C of phenyl), 116.71 (CH of phenyl), 158.49 ( $\mathrm{C}$ of phenyl), 158.58 ( $\mathrm{C}$ of phenyl), 118.28 ( $\mathrm{CH}$ of phenyl), 124.15 ( $\mathrm{CH}$ of phenyl), 122.53 ( $\mathrm{CH}$ of ethylene), 40.04 ( $\mathrm{CH}$, diazepane), 32.63 
( $\mathrm{CH}_{2}$, diazepane), $24.04\left(\mathrm{CH}_{2}\right.$, diazepane $), 24.11\left(\mathrm{CH}_{2}\right.$ of $\left.\mathrm{COC}_{2} \mathrm{H}_{5}\right) ; \mathrm{MS}$ ES + (ToF): m/z 378.4.

\section{4-Benzoyl-N-hydroxy-1-(3-(3,4-dihydroxyphenyl) acryloyl)-1,4-diazepane-2-carboxamide (15)}

Mp $\left({ }^{\circ} \mathrm{C}\right)$ 240-242; Yield-69.3\%; IR ( $\mathrm{KBr}$ pellets, $\mathrm{cm}^{-1}$ ): $1168(\mathrm{C}-\mathrm{O}), 1205(\mathrm{C}-\mathrm{N}), 1263(\mathrm{C}-\mathrm{H}), 1323(\mathrm{C}=\mathrm{C}), 1394$ $(\mathrm{C}=\mathrm{N}), 1440(\mathrm{C}=\mathrm{O}), 1469(\mathrm{C} \equiv \mathrm{C}), 1581(\mathrm{C} \equiv \mathrm{N}), 1637(-$ $\mathrm{C}=\mathrm{O}-\mathrm{OH}), 1728(\mathrm{C}-\mathrm{H}), 1843(=\mathrm{C}-\mathrm{H}), 1865(\equiv \mathrm{C}-\mathrm{H})$, $2133(\equiv \mathrm{C}-\mathrm{H}), 2243(\mathrm{~N}-\mathrm{H}), 2306(\mathrm{~N}-\mathrm{H}), 2353(\mathrm{O}-\mathrm{H})$, $2490(\mathrm{O}-\mathrm{H}) ;{ }^{1} \mathrm{H}$ NMR (DMSO- $\left.d_{6}, \delta \mathrm{ppm}\right): 2.197(\mathrm{~s}, 1 \mathrm{H}$, $\mathrm{OH}$ of $\mathrm{NHOH}), 8.123(\mathrm{~s}, 1 \mathrm{H}, \mathrm{NH}$ of $\mathrm{CONHOH}), 4.731$ (d, 2H, aromatic $\mathrm{OH}), 2.780-5.012(\mathrm{~m}, 9 \mathrm{H}$, diazepane), 6.945-7.535 (m, 8H, CH, aromatic), 7.334 (s, $1 \mathrm{H}, \mathrm{CH}$ of ethylene), 6.985 (s, $1 \mathrm{H}, \mathrm{CH}$ of ethylene); ${ }^{13} \mathrm{CNMR}$ (DMSO- $\left.d_{6}, \delta \mathrm{ppm}\right): 168.25\left(\mathrm{C}=\mathrm{O}\right.$ of $\left.\mathrm{COC}_{6} \mathrm{H}_{5}\right), 167.72$ ( $\mathrm{C}=\mathrm{O}$ of $\mathrm{CONHOH}), 158.58(\mathrm{C}=\mathrm{O}$ of amide), 138.08 ( $\mathrm{CH}$ of ethylene), 130.82 (C of phenyl), $116.71(\mathrm{CH}$ of phenyl), 158.49 ( $\mathrm{CH}$ of phenyl), 158.58 (C of phenyl), $118.28(\mathrm{CH}$ of phenyl), $122.53(\mathrm{CH}$ of phenyl), 124.15 ( $\mathrm{CH}$ of ethylene), $40.04(\mathrm{CH}$, diazepane); $\mathrm{MS} \mathrm{ES}+(\mathrm{ToF})$ : $\mathrm{m} / \mathrm{z} 427.5$.

\section{4-Butyryl-N-hydroxy-1-(3-(3,4-dihydroxyphenyl) acryloyl)-1,4-diazepane-2-carboxamide (16)}

$\mathrm{Mp}\left({ }^{\circ} \mathrm{C}\right)$ 266; Yield-68.9\%; IR (KBr pellets, $\left.\mathrm{cm}^{-1}\right): 1163$ (C-O), $1296(-\mathrm{C}-\mathrm{N}), 1440(-\mathrm{C}-\mathrm{H}), 1581(\mathrm{C}=\mathrm{C}), 1637$ $(\mathrm{C}=\mathrm{N}), 1705(\mathrm{C}=\mathrm{O}), 1716(\mathrm{C}=\mathrm{O}), 2133(\mathrm{C} \equiv \mathrm{C}), 2245$ $(\mathrm{C} \equiv \mathrm{N}), 2675(-\mathrm{C}-\mathrm{H}=\mathrm{O}), 2744(-\mathrm{C}-\mathrm{H}=\mathrm{O}), 2935(\mathrm{C}-\mathrm{H})$, $2970(=\mathrm{C}-\mathrm{H}), 3064(=\mathrm{C}-\mathrm{H}), 3246(\equiv \mathrm{C}-\mathrm{H}), 3408(\mathrm{~N}-\mathrm{H})$, $3755(\mathrm{O}-\mathrm{H}) ;{ }^{1} \mathrm{H}$ NMR (DMSO- $\left.d_{6}, \delta \mathrm{ppm}\right): 2.010(\mathrm{~s}, 1 \mathrm{H}$, $\mathrm{OH}$ of $\mathrm{NHOH}), 8.110(\mathrm{~s}, 1 \mathrm{H}, \mathrm{NH}$ of $\mathrm{CONHOH}), 4.910$ (d, $2 \mathrm{H}$, aromatic $\mathrm{OH}), 1.171-5.161(\mathrm{~m}, 9 \mathrm{H}$, diazepane), 6.981-7.523 (m, 3H, CH, aromatic), $7.334(\mathrm{~s}, 1 \mathrm{H}, \mathrm{CH}$ of ethylene), 7.217 (s, $1 \mathrm{H}, \mathrm{CH}$ of ethylene); ${ }^{13} \mathrm{CNMR}$ (DMSO- $\left.d_{6}, \delta \mathrm{ppm}\right): 168.25\left(\mathrm{C}=\mathrm{O}\right.$ of $\left.\mathrm{COC}_{3} \mathrm{H}_{7}\right), 167.72$ ( $\mathrm{C}=\mathrm{O}$ of $\mathrm{CONHOH}), 158.58(\mathrm{C}=\mathrm{O}$ of amide), 138.08 ( $\mathrm{CH}$ of ethylene), 130.82 (C of phenyl), $116.71(\mathrm{CH}$ of phenyl), 158.49 ( $\mathrm{CH}$ of phenyl), 158.58 ( $\mathrm{C}$ of phenyl), $118.28(\mathrm{CH}$ of phenyl), $122.53(\mathrm{CH}$ of phenyl), 122.53 (CH of ethylene), 40.04 $\left(\mathrm{CH}\right.$, diazepane), $24.04\left(\mathrm{CH}_{2}\right.$, diazepane), $24.11\left(\mathrm{CH}_{2}\right.$ of $\left.\mathrm{COC}_{3} \mathrm{H}_{7}\right)$.

\section{Biological evaluation Cell culture}

Dulbecco's Modified Eagle Medium (DMEM), Penicillin and Streptomycin purchased from Himedia, Mumbai; Fetal Calf Serum (FCS) from Lonza, Belgium; DMSO (dimethyl sulfoxide) from Sigma-Aldrich, USA; MTT reagent [3-(4,5-dimethylthiazol-2-yl)-2, 5-diphenyltetrazolium bromide] from Merck, India and Paclitaxel from Dabur, India. Cell lines were procured from National Centre for Cell Science (NCCS), Pune, India.
A549 human lung cancer cells were grown in DMEM supplemented with $100 \mathrm{U} / \mathrm{mL}$, Penicillin G, $100 \mathrm{lg} / \mathrm{mL}$ Streptomycin, $0.25 \mathrm{lg} / \mathrm{mL}$, Amphotericin, and 10\% heat inactivated fetal bovine serum. Cultures were maintained at $37^{\circ} \mathrm{C}$ in a $5 \% \mathrm{CO} 2,95 \%$ air atmosphere.

\section{In vitro anticancer assays}

MTT assay Preliminary cytotoxic activity was assayed by MTT assay as previously described [26]. In brief, A549 cell lines were grown for $48 \mathrm{~h}$ after incubation at various concentrations of synthesized compounds. The optical density (OD) was measured by ELISA plate reader at $570 \mathrm{~nm}$ with a reference wavelength of $630 \mathrm{~nm}$. OD was expressed as percentage cell survival (absorbance of treated wells/absorbance of control wells $\times 100$ ). Results were expressed as Mean \pm S.E. and based on the results; the active compounds were considered to be significant which gave less than $50 \%$ survival at the exposure time of $48 \mathrm{~h}$.

Semi-quantitative RT-PCR (mRNA analysis) Based on preliminary cytotoxicity results, gene expression was assayed by semi-quantitative RT-PCR as previously described [37]. In short, cancer cells $\left(2 \times 10^{6}\right.$ cells $\left./ \mathrm{mL}\right)$ were treated with selected active compounds for $18 \mathrm{~h}$ followed by isolation of total RNA and then quantification. After that, $1 \mu \mathrm{g}$ of total RNA was used for cDNA synthesis. The cDNA amplification was done with gene specific primers: "human MMP-2 (5'-GTG CTG AAG GAC ACA CTA AAG AAG A-3' ${ }^{\prime}, 3^{\prime}$-GGA TGT TGA AAC TCT TCC TAC CGT T-5'); MMP-9 (5'-CAC TGT CCA CCC CTC AGA GC-3' ${ }^{\prime}, 3^{\prime}$-GGA ATA GCG GCT GTT CAC CG-5'), $\beta$-actin ( $5^{\prime}$-TGT GAT GGT GGG AAT GGG TCA G-3', $5^{\prime}$-TTT GAT GTC ACG CAC GAT TTC C-3'). $\beta$-Actin primers were used as normalization control. The PCR products were separated on a $2 \%$ agarose gel containing ethidium bromide $(0.5 \mu \mathrm{g} / \mathrm{mL})$, visualized, and photographed using a gel documentation system.

\section{Molecular docking studies}

The docking studies were performed for selected compounds $(6,7,8,15$ and 16$)$ in the binding site of MMP-2 and MMP-9 proteins (PDB entries: $1 \mathrm{HOV}$ and $4 \mathrm{H} 3 \mathrm{X}$ respectively) using AutoDock Vina [23, 32] and graphical user interface, Auto-dock tools installed on windows 7 [17]. The X-ray crystallographic information of MMP-2 and MMP-9 proteins was acquired from protein data bank (http://www.rcsb.org/pdb) and after evaluation of a number of entries, the best X-ray structures were chosen by analyzing the proteins with the highest resolution. The PDB file of MMP-2 and MMP-9 proteins was edited with the help of PyMOL, and $\alpha$ chain was removed along with the complexed inhibitor. All interacting ions and water 
molecules were removed. The PDBQT file for the proteins was generated with the help of AutoDock tools by addition of all polar hydrogen atoms charge assignment to the macromolecule. The geometries of the ligands were optimized by Open Babel using force field [20]. The ligands were prepared for docking by using AutoDock tools by assigning the charges to all the atoms and storing them as PDBQT file. The calculations of grid parameters were accomplished by using the Grid tool in Auto-Dock tools. The grid parameter file possessing all information regarding the protein, size of grid, geometry of search space and ligand was built and was kept as 'Conf.txt.'

The docking of co-crystallized inhibitors into the active site of target proteins was executed for the determination of accuracy of docking protocol. The optimized ligand molecules in PDBQT format were docked in the active site of MMP-2 and MMP-9 proteins by means of AutoDock Vina. Docking runs were launched from the command line, followed by the generation and scoring of best nine poses, for each and every ligand using AutoDock Vina scoring function. At the end of the docking, ligands with utmost favorable free energy $(-\Delta G)$ of binding were carefully chosen. "Lower is the value; higher is the interaction, thus, stability of the ligand-protein complex". The hydrophobic, hydrogen bond and other interactions were further analyzed for the docked ligands by using PyMOL and best poses in the binding site were drawn.

\section{Results}

\section{Chemistry}

The substituted cinnamic acid derivatives were synthesized by the synthetic route as highlighted in Fig. 2. In the first step, substituted benzaldehyde derivatives and malonic acid were reacted to form cinnamic acid derivatives. In the second step, the corresponding cinnamic acid derivatives were reacted with tert-butyl (3-aminopropyl)carbamate to synthesize tert-butyl (3-cinnamamidopropyl)carbamate derivatives. The tert-butyl (3-cinnamamidopropyl)carbamate derivatives were reacted with 2,3-dibromopropanoic acid and potassium carbonate using a microwave synthesizer (Temp. $120^{\circ} \mathrm{C}, 90 \mathrm{~W}$ Power, and 20 min reaction time) resulting in 4-(tert-butoxycarbonyl)-1-cinnamoyl-1,4-diazepane2-carboxylic acid derivatives. This step was followed by reaction with acyl and aryl chlorides to obtain diazepine substituted cinnamic acid derivatives. In the last step the diazepine substituted cinnamic acid derivatives were reacted with hydroxylamine to get the desired molecules. The physicochemical characteristics of the synthesized compounds are presented in Table 1 . The synthesized compounds were characterized by FTIR, ${ }^{1} \mathrm{H}$ and ${ }^{13} \mathrm{C}$ NMR, and Mass spectra and the results were in accord with the allocated molecular structures.

\section{MTT assay}

The anticancer potential of the synthesized compounds was measured (1-16) by MTT assay. The results indicated that all the synthesized compounds were found to be active against A549 cancer cells and showed dose-dependent cytotoxicity. Data also pointed out that amongst the synthesized compounds; compounds $8(\mathrm{IC} 50=7.7 \pm 0.5 \mu \mathrm{M})$ and $\mathbf{1 6}(\mathrm{IC} 50=7.3 \pm 0.3 \mu \mathrm{M})$ showed comparable cytotoxicity in comparison with standard (paclitaxel-IC50 =7.3 $\pm 0.7 \mu \mathrm{M}$ ) $[2,6,9,15$, $21,35,38]$. Compounds 7 (IC50 $=8.5 \pm 0.8 \mu \mathrm{M})$, and $\mathbf{1 5}$ $(8.2 \pm 0.7 \mu \mathrm{M})$ also showed considerable activity against the cancer cell lines (Table 1). Compound $\mathbf{1 6}$ was found to be most potent against A549 cells.

\section{Selected compounds downregulates the expressions of MMPs (-2 and -9)}

MMPs (-2 and -9$)$ have been indicated to be associated with cancer metastasis, we, therefore, investigated whether the compounds 7, 8, 15 and 16 were involved in invasion down regulation. It was confirmed by the inhibition of MMPs activity by RT-PCR (m-RNA analysis) method by measuring the expression levels of MMP-2 and MMP-9. We found that compounds 8 and 16 significantly inhibited MMP-2 and MMP-9 activity in A549 cells; however the inhibition of MMP-2 and MMP-9 activity by compounds 7 and 15 was comparatively less. Compounds 8 and 16 significantly suppressed the expression of MMP-2 and MMP-9 protein and mRNA levels (Fig. 3a, b) which forms a specific complex with the MMPs and thus inhibits the activation of MMP-2 and MMP-9. The results indicated that compounds 8 and 16 have the tendency to inhibit the metastasis of cancer. Based on the results, it can be concluded that compound $\mathbf{1 6}$ may be taken as a lead compound for the discovery of new drug molecules for the treatment of lung cancer.

\section{Molecular docking}

Lead optimization of the synthesized compounds was done by computation of drug-likeness properties molecular weight, partition coefficient i.e., $\log$ P, hydrogen bond donors (HBA), and hydrogen bond acceptors (HBD). Most of the selected compounds for in silico studies were found to possess drug like properties as derived by Lipinski's rule of five. Docking studies were carried out to evaluate the affinity and binding interactions of the selected synthesized molecules in the active site of MMP-2 (PDB entry: 1HOV) and MMP-9 (PDB entry: 4H3X) proteins using AutoDock Vina and the graphical user interface, AutoDockTools installed on Windows 7. The docking protocol was validated by docking of co-crystallized ligand into the active site, and the resulting binding pose was compared with 
<smiles>[R]c1ccc(C(=O)[CH-][CH]C(=O)O)cc1[R]</smiles><smiles>[R]c1ccc(/C=C/C(=O)N2CCCN(C(=O)/C=C/c3ccc([R])c([R])c3)C(C(=O)O)C2)cc1[R]</smiles><smiles>[R]c1ccc(/C=C/C(=O)N2CCCN(C(=O)OC(C)=O)CC2C(=O)OC)cc1[R]</smiles><smiles>[AlH]Cl</smiles><smiles>[R]c1ccc(/C=C/C(=O)N2CCCN([Y])CC2C(=O)NO)cc1/C=C/C(=O)N1CCCN([Y])CC1C(=O)OC</smiles>

Fig. 2 Synthesis of diazepine substituted cinnamic acid derivatives

that of reference ligands (MMP-2: $N-\{4-[(1$-hydroxycarbamoyl-2-methyl-propyl)-(2-morpholin4-yl-ethyl)-sulfamoyl]-4-pentyl-benzamide; MMP-9: $N$-2-(biphenyl-4-ylsulfonyl)- $N$-2-(isopropyloxy)-acetohydroxamic acid). The ligands had a similar binding pattern and superposition to that of co-crystallized ligands, thus validating the docking protocol. The selected compounds showed appreciable binding to the binding site of MMP-2 and MMP-9 proteins as determined by analyzing their bonding interactions in terms of $\mathrm{H}$-bond, hydrophobic interactions and binding free energy $(-\Delta \mathrm{G}, \mathrm{kcal} /$ mol) of the selected best docked poses (Table 2). These compounds were further analyzed in details by Molecular Visualization Tool, PyMOL.

\section{MMP-2 overlays}

MMP-2 overlays The overlay of docked poses of compounds $7,8,15$ and 16 with that of $1 \mathrm{HOV}$ ligand showed that compounds $7,8,15$ and 16 had similar binding pattern in the active site of MMP-2 protein as that of co-crystallized inhibitor (Figs. 4a, 5a, 6a and 7a). The docked pose of compound 7 showed the H-bond interaction between
$\mathrm{NH}$ of $\mathrm{NHOH}$ and carbonyl group with $\mathrm{COOH}$ of Glu121 residue and $\mathrm{NH}$ of Leu83 residue in the active site of MMP-2 protein with H-bond distances of 3.3 and $4.3 \AA$, respectively (Fig. 4b). The docked pose of compound 8 showed the $\mathrm{H}$-bond interaction between carbonyl with $\mathrm{NH}$ of Leu83 and Ala84 residues (3.0 and $3.7 \AA$ ); and between $\mathrm{NH}$ of $\mathrm{NHOH}$ and $\mathrm{COOH}$ of Glu121 residue (2.8 $\AA$ ) (Fig. 5b). The docked pose of compound 15 showed the $\mathrm{H}$-bond interaction between carbonyl with $\mathrm{NH}$ of Leu83 and Ala84 residues (3.4 and $3.5 \AA$ ); and between $\mathrm{NH}$ of $\mathrm{NHOH}$ and $\mathrm{COOH}$ of Glu121 residue (3.3 $\AA$ ) (Fig. 6b). The docked pose of compound $\mathbf{1 6}$ showed the $\mathrm{H}$-bond interaction between carbonyl with $\mathrm{NH}$ of Leu83 and Ala84 residues (2.9 and 3.4 $\AA$ ); and between $\mathrm{NH}$ of $\mathrm{NHOH}$ and $\mathrm{COOH}$ of Glu121 residue (2.8 $\AA$ ) (Fig. 7b). All the selected compounds showed appreciable metal interaction between $\mathrm{OH}$ of $\mathrm{NHOH}$ and $\mathrm{Zn}^{2+}$ ion in the binding site of MMP-2 protein.

MMP-9 overlays The overlay of docked poses of compounds 7, 8, 15 and 16 with that of 4H3X ligand showed that the selected compounds had the similar binding pat- 
Table 1 Physiochemical properties of synthesized diazepine substituted cinnamic acid derivatives and cytotoxicity evaluation on A549 cell lines

\begin{tabular}{|c|c|c|c|c|c|c|}
\hline C. no. & R1, R2 & $Y$ & Mol. wt & Rf value ${ }^{a}$ & M. Pt. & $I C_{50}(\mu M)^{b}$ \\
\hline 1 & $\mathrm{H}, \mathrm{H}$ & $-\mathrm{COC}_{6} \mathrm{H}_{5}$ & 393.443 & 0.57 & 210 & $13.1 \pm 0.2$ \\
\hline 2 & $\mathrm{H}, \mathrm{H}$ & $-\mathrm{COCH}_{2} \mathrm{CH}_{2} \mathrm{CH}_{3}$ & 359.426 & 0.51 & $121-123$ & $12.5 \pm 0.9$ \\
\hline 3 & $\mathrm{H}, \mathrm{H}$ & $-\mathrm{COCH}_{3}$ & 331.372 & 0.46 & 164 & $17.8 \pm 0.5$ \\
\hline 4 & $\mathrm{H}, \mathrm{H}$ & $-\mathrm{COCH}_{2} \mathrm{CH}_{3}$ & 345.399 & 0.55 & 190 & $13.5 \pm 0.7$ \\
\hline 5 & $3-\mathrm{OH}$ & $-\mathrm{COCH}_{3}$ & 347.371 & 0.47 & 123 & $13.9 \pm 0.6$ \\
\hline 6 & $3-\mathrm{OH}$ & $-\mathrm{COCH}_{2} \mathrm{CH}_{3}$ & 361.398 & 0.52 & $125-127$ & $11.2 \pm 0.6$ \\
\hline 7 & $3-\mathrm{OH}$ & $-\mathrm{COC}_{6} \mathrm{H}_{5}$ & 409.442 & 0.50 & $230-231.5$ & $8.5 \pm 0.8$ \\
\hline 8 & $3-\mathrm{OH}$ & $-\mathrm{COCH}_{2} \mathrm{CH}_{2} \mathrm{CH}_{3}$ & 375.425 & 0.60 & $255-255.3$ & $7.7 \pm 0.5$ \\
\hline 9 & $4-\mathrm{OH}$ & $-\mathrm{COCH}_{3}$ & 347.371 & 0.56 & $124-125$ & $13.6 \pm 0.4$ \\
\hline 10 & $4-\mathrm{OH}$ & $-\mathrm{COCH}_{2} \mathrm{CH}_{3}$ & 361.398 & 0.80 & 135 & $12.8 \pm 0.9$ \\
\hline 11 & $4-\mathrm{OH}$ & $-\mathrm{COC}_{6} \mathrm{H}_{5}$ & 409.442 & 0.48 & $232-233$ & $11.1 \pm 0.7$ \\
\hline 12 & $4-\mathrm{OH}$ & $-\mathrm{COCH}_{2} \mathrm{CH}_{2} \mathrm{CH}_{3}$ & 375.425 & 0.30 & $251-252$ & $10.3 \pm 0.7$ \\
\hline 13 & 3, 4-di OH & $-\mathrm{COCH}_{3}$ & 363.370 & 0.75 & 180 & $11.8 \pm 0.6$ \\
\hline 14 & 3, 4-di OH & $-\mathrm{COCH}_{2} \mathrm{CH}_{3}$ & 377.397 & 0.40 & 200 & $10.1 \pm 0.5$ \\
\hline 15 & 3,4-di OH & $-\mathrm{COC}_{6} \mathrm{H}_{5}$ & 425.441 & 0.65 & $240-242$ & $8.2 \pm 0.7$ \\
\hline 16 & 3, 4-di OH & $-\mathrm{COCH}_{2} \mathrm{CH}_{2} \mathrm{CH}_{3}$ & 391.424 & 0.75 & 266 & $7.3 \pm 0.3$ \\
\hline $\mathrm{Pac}^{\mathrm{c}}$ & - & - & - & - & - & $7.3 \pm 0.7$ \\
\hline
\end{tabular}

a Mobile phase: dichloromethane: methanol (19:1)

b Mean \pm S.D. $(n=3)$

c Pac-Paclitaxel
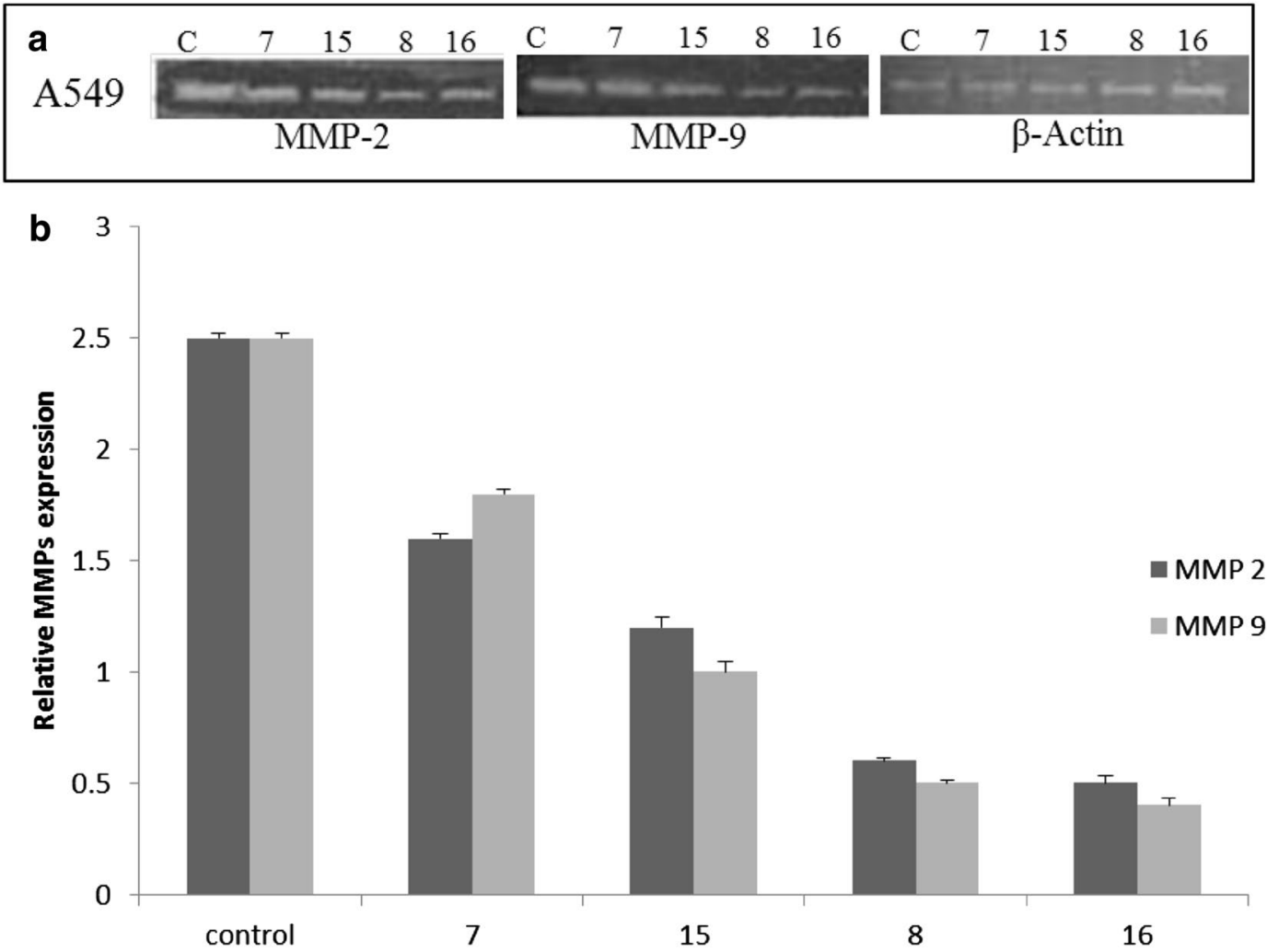

Fig. 3 a, b Relative mRNA expression of gelatinases MMPs shows a down regulation in collagen degrading enzymes upon treatment. c Control; Compounds $7(8.5 \mu \mathrm{M}), 8(7.7 \mu \mathrm{M}), 15(8.2 \mu \mathrm{M})$, and $16(7.3 \mu \mathrm{M})$. Data are illustrative of a minimum of three independent experiments 
tern in the active site of MMP-9 protein as that of co-crystallized inhibitor (Figs. 8a, 9a, 10a and 11a). The docked pose of compound 7 showed a weak $\mathrm{H}$-bond interaction between carbonyl and $\mathrm{NH}$ of Ala189 residue in the active site of MMP-9 protein (Fig. 8b). The docked pose of compound 8 showed a weak $\mathrm{H}$-bond interaction between carbonyl and $\mathrm{NH}$ of Leu188 residue in the active site of MMP-9 protein (Fig. 9b). The docked pose of compound
15 showed appreciable $\mathrm{H}$-bond interactions between carbonyl and NH of Leu188 and Ala189residues in the active site of MMP-9 protein (H-bond distance of 3.2 and $4.7 \AA$ respectively) (Fig. 10b). The docked pose of compound $\mathbf{1 6}$ showed a weak $\mathrm{H}$-bond between carbonyl group and $\mathrm{NH}$ of Leu188 residue (Fig. 11b). The hydroxamate group of all the docked compounds showed appreciable metal interaction with $\mathrm{Zn}^{2+}$ of the MMP-9 protein.

Table 2 Docking scores and molecular properties of selected compounds

\begin{tabular}{|c|c|c|c|c|c|c|}
\hline Compound & Mol. wt ${ }^{a}$ & $\mathrm{HBA}^{\mathrm{a}}$ & $\mathrm{HBD}^{\mathrm{a}}$ & $\log P^{a}$ & $\Delta \mathrm{G}(\mathrm{MMP}-2)^{*}$ & $\Delta \mathrm{G}(\mathrm{MMP}-9)^{*}$ \\
\hline 7 & 409.44 & 5 & 3 & 1.29 & -7.8 & -8.0 \\
\hline 8 & 375.42 & 5 & 3 & 0.59 & -7.0 & -7.6 \\
\hline 15 & 425.44 & 6 & 4 & 1.34 & -8.1 & -8.9 \\
\hline 16 & 391.42 & 6 & 4 & 0.75 & -7.0 & -7.7 \\
\hline
\end{tabular}

${ }^{*} \Delta \mathrm{G}(\mathrm{KJ} / \mathrm{mol})$ for reference ligand: -8.3 and -8.5 for MMP-2 and MMP-9, respectively

a Mol. wt, HBA, HBD, and log P were calculated by Marvin tools of Marvin Sketch
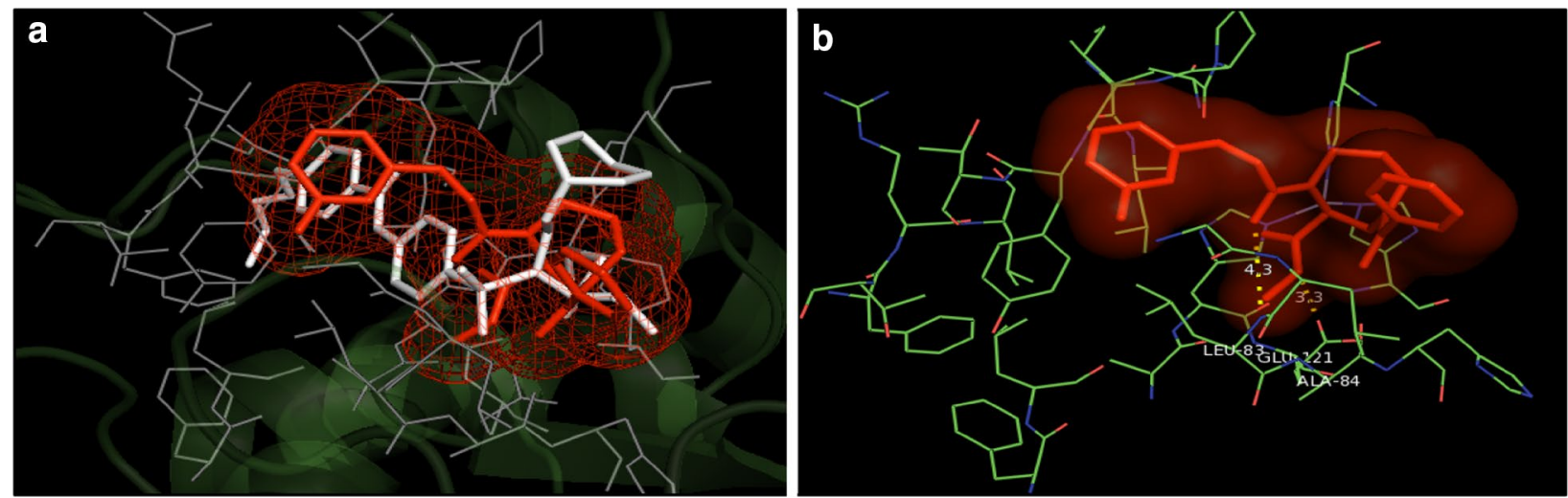

Fig. 4 a Overlay of the docked pose of compound $\mathbf{7}$ (red) with that of PDB Ligand 1HOV (white); $\mathbf{b}$ docked pose: H-bond interaction of compound $\mathbf{7}$ in the binding site of MMP-2 protein
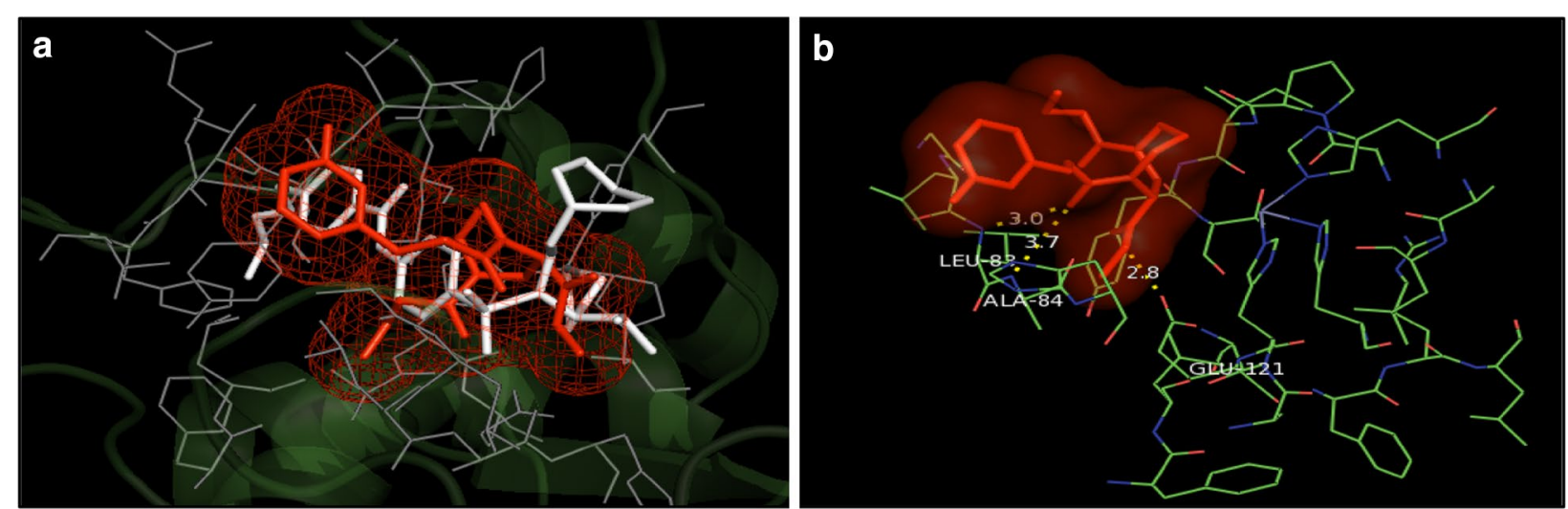

Fig. 5 a Overlay of the docked pose of compound $\mathbf{8}$ (red) with that of PDB Ligand 1HOV (white); b docked pose: H-bond interaction of compound $\mathbf{8}$ in the binding site of MMP-2 protein 

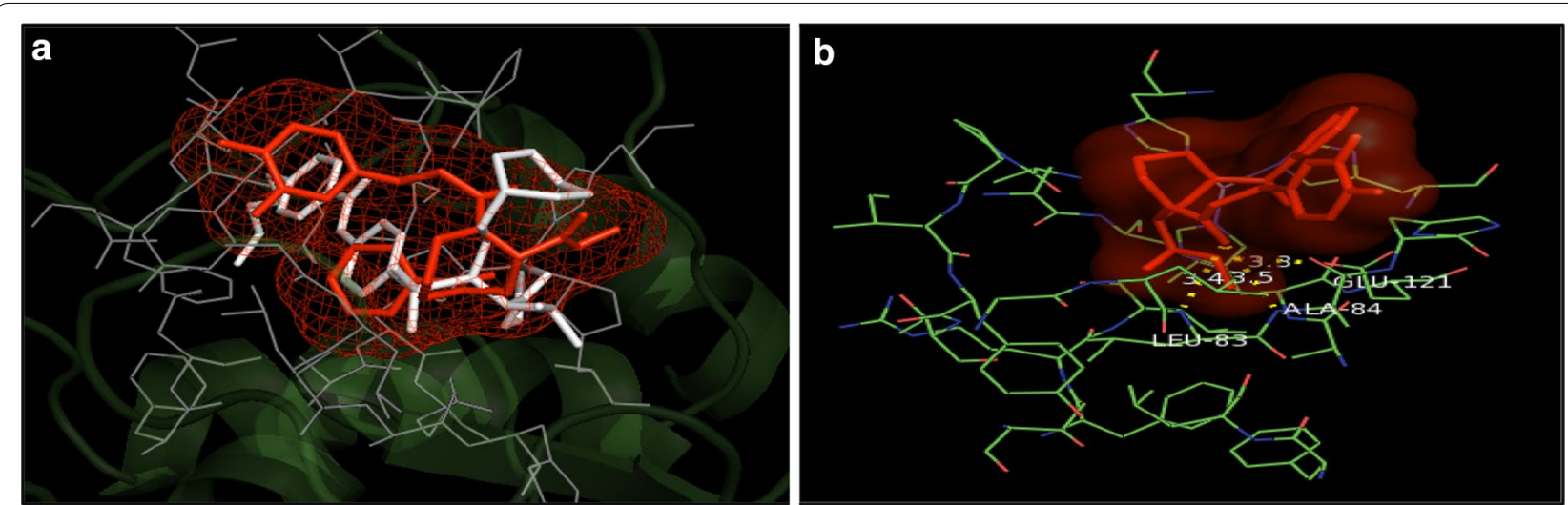

Fig. 6 a Overlay of the docked pose of compound $\mathbf{1 5}$ (red) with that of PDB Ligand 1HOV (white); b docked pose: H-bond interaction of compound $\mathbf{1 5}$ in the binding site of MMP-2 protein
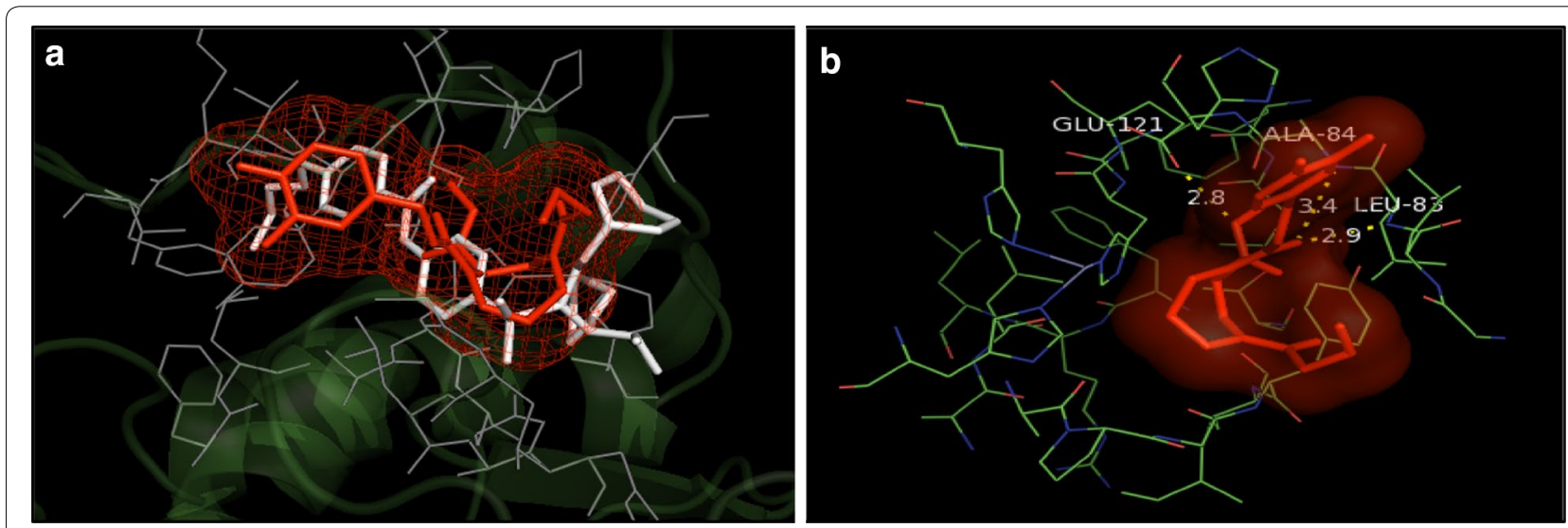

Fig. 7 a Overlay of the docked pose of compound $\mathbf{1 6}$ (red) with that of PDB Ligand 1HOV (white); $\mathbf{b}$ docked pose: H-bond interaction of compound $\mathbf{1 6}$ in the binding site of MMP-2 protein

\section{Discussion}

Metastasis is the leading reason for the resultant mortality of patients with cancer and is a major reason for treatment failure [13]. Previous reports have demonstrated that diazepine and caffeic acid (hydroxycinnamic acid) are correlated with MMPs inhibitory activity, however, there are no studies addressing anti-metastasis activity of diazepine substituted cinnamic acid derivatives. In the current study, a series of cinnamic acid derivatives clubbed with diazepine ring has been synthesized and characterized by physicochemical properties and spectral techniques. The synthesized compounds were screened for their in vitro anticancer potential and the results of cytotoxicity studies revealed that all the synthesized compounds were active against A549 cancer cell lines. Compounds 8 (IC50 $7.7 \mu \mathrm{M})$ and $16(\mathrm{IC} 50=7.3 \mu \mathrm{M})$ were found to be the most potent against the cancer lines. Further, it was proved that compounds 8 and 16 significantly suppressed the expression of MMP-2 and MMP-9 protein and mRNA levels which forms a specific complex with the MMPs and thus inhibits the activation of MMP-2 and MMP-9. The results indicated that compounds 8 and 16 have the tendency to inhibit the invasion and metastasis of cancer. Based on the results, it can be concluded that compound $\mathbf{1 6}$ may be taken as a lead compound for the discovery of new drug molecules for the treatment of lung cancer.

Based on the anticancer studies of synthesized diazepine substituted cinnamic acid derivatives, the following SAR can be concluded upon. Compounds 1-4 without hydroxyl groups (either 3 or 4 position) on the cinnamic acid showed least activity. This confirms the requirement of $-\mathrm{OH}$ groups at these positions. The presence of $-\mathrm{OH}$ groups at either 3 or 4 position of cinnamic acid increased the anticancer activity as see in case of compounds 5-16. Further the compounds having di-OH 


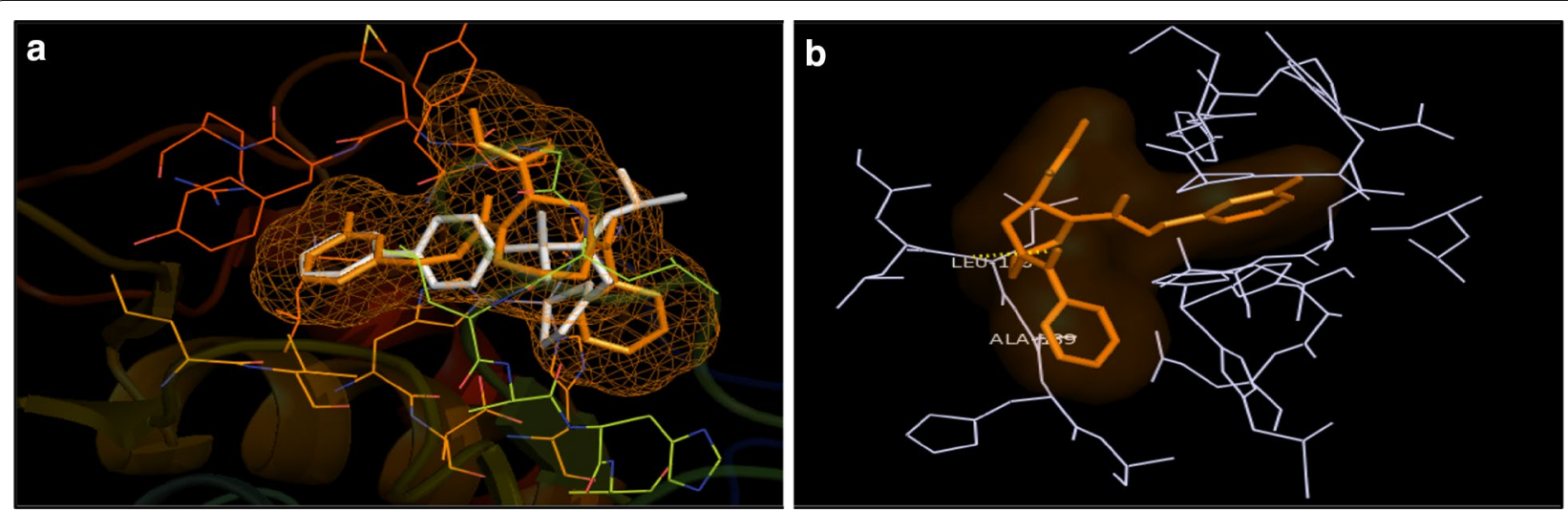

Fig. 8 a Overlay of the docked pose of compound $\mathbf{7}$ (orange) with that of PDB Ligand 4H3X (white); b docked pose: H-bond interaction of compound $\mathbf{7}$ in the binding site of MMP-9 protein
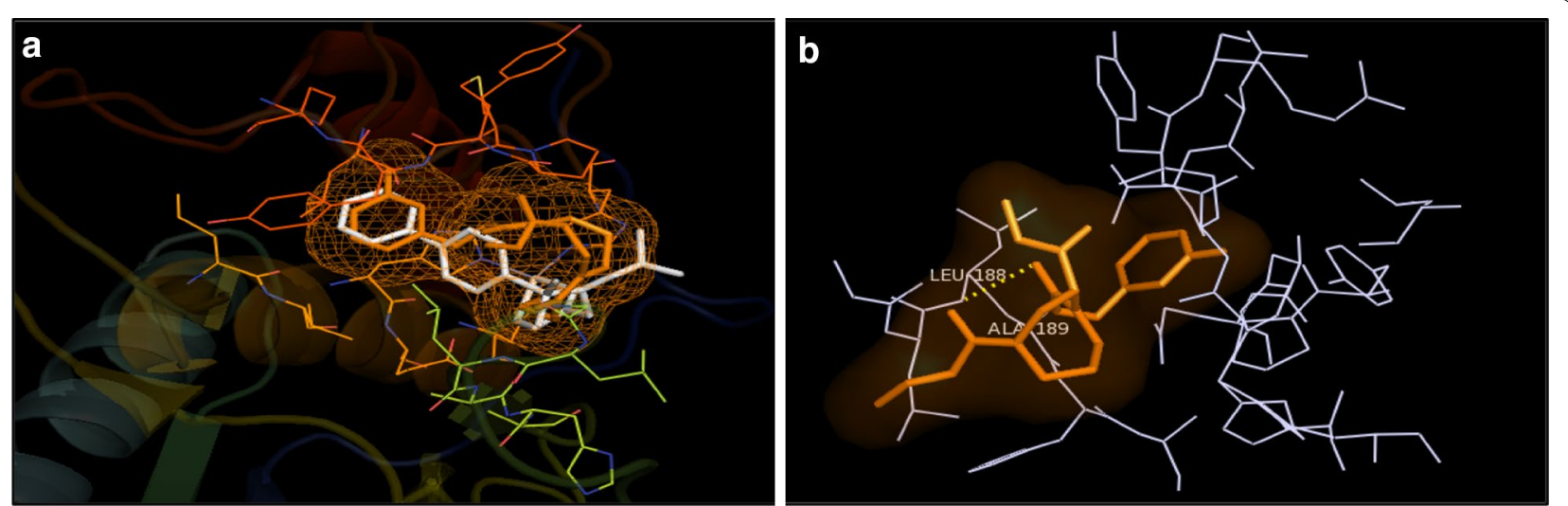

Fig. 9 a Overlay of the docked pose of compound $\mathbf{8}$ (orange) with that of PDB Ligand 4H3X (white); b docked pose: H-bond interaction of compound $\mathbf{8}$ in the binding site of MMP-9 protein
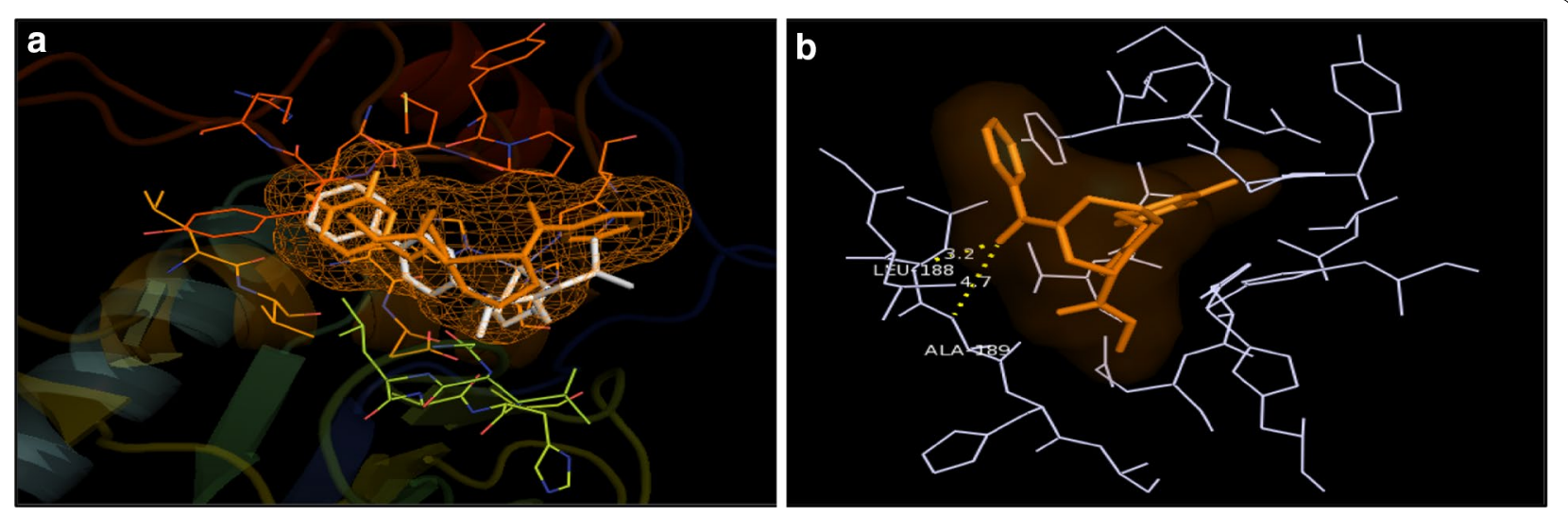

Fig. 10 a Overlay of the docked pose of compound $\mathbf{1 5}$ (orange) with that of PDB Ligand 4H3X (white); $\mathbf{b}$ docked pose: H-bond interaction of compound $\mathbf{1 5}$ in the binding site of MMP-9 protein 

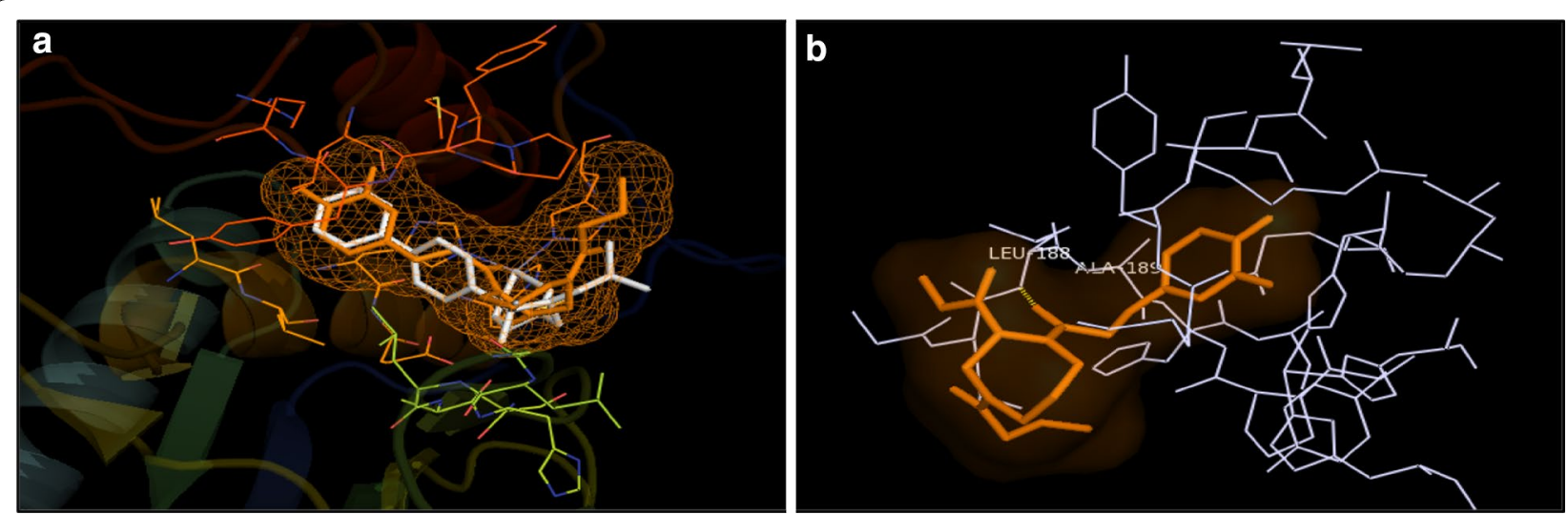

Fig. 11 a Overlay of the docked pose of compound $\mathbf{1 6}$ (orange) with that of PDB Ligand 4H3X (white); b docked pose: H-bond interaction of compound $\mathbf{1 6}$ in the binding site of MMP-9 protein

groups at both 3,4 positions showed the most potent anticancer activity as observed in 16 and significantly inhibited the expression of MMPs and also decreased the invasive potential of A549 cells. These results showed that both the -OH groups were important for MMP inhibition and selectivity. The possible reason for this might be the solvent exposed region in the MMPs, which results in better binding. In addition, the presence of larger and extended groups like isobutyl group at $\mathrm{N}$ on 4-position of diazepine ring (i.e. the P1 site) significantly enhanced the anticancer activity and further the MMP inhibition and selectivity as in case of compounds 8 and 16 . This is due to the fact that hydrophobic groups have strong hydrophobic interactions with MMP proteins.

In addition, the mode of interactions between compounds 7, 8, 15 and 16 with MMP-2 and MMP-9 were investigated through molecular docking studies and to find out a relationship between the anticancer properties of synthesized compounds and their structural features. Molecular docking study revealed that compounds $7,8,15$ and 16 directly interacted with active site residues to inhibit MMP-2 and MMP-9 activities. There is evidence that MMP-2 has a channel-like S1' cavity and MMP-9 has a pocket-like S1' cavity with a floorboard [1, 12,30 ] and the S1' is a substrate binding pocket, formed by two subdomains. S1' pocket is found in the center of active site cleft neighbouring to active site zinc ion and this pocket consists of Asp 185-Leu 188 and Pro 421Tyr 423 which are accountable for hydrogen bonding to substrates/inhibitors. The hedge of S1' cavity is formed by side chains of Leu 188, Leu 397, Val 398, His 401, Leu 418, and Met 422-Tyr 423 as main chain. Leu 397 and Val 398 are specific to MMP-9 [30]. Compounds 7, 8, 15 and 16 overlays showed similar binding pattern in the active site of MMP-2 protein as that of co-crystallized inhibitor.
Further, docked poses of compounds 7, 8, 15 and 16, showed $\mathrm{H}$-bond interaction: between $\mathrm{NH}$ of $\mathrm{NHOH}$ and carbonyl group with $\mathrm{COOH}$ of Glu 121 residue and $\mathrm{NH}$ of Leu 83residue (compound 7); between carbonyl with $\mathrm{NH}$ of Leu 83 and Ala 84 residues and between $\mathrm{NH}$ of $\mathrm{NHOH}$ and $\mathrm{COOH}$ of Glu 121 (Compound 8); between carbonyl with $\mathrm{NH}$ of Leu 83 and Ala 84and between $\mathrm{NH}$ of $\mathrm{NHOH}$ and $\mathrm{COOH}$ of Glu 121residue (compound 15); between carbonyl with $\mathrm{NH}$ of Leu 83 and Ala 84 and between $\mathrm{NH}$ of $\mathrm{NHOH}$ and $\mathrm{COOH}$ of Glu 121 residues (compound 16) in the active site of MMP-2 protein, respectively. All the selected compounds showed appreciable metal interaction between $\mathrm{OH}$ of $\mathrm{NHOH}$ and $\mathrm{Zn}^{2+}$ ion in the binding site of MMP-2 protein.

Further, the docked pose of compounds 7, 8, 15 and 16 showed $\mathrm{H}$-bond interaction between $\mathrm{H}$-bond interaction between carbonyl and $\mathrm{NH}$ of Ala189 residue (compound 7), carbonyl and $\mathrm{NH}$ of Leu 188 residue (compound 8); between carbonyl and NH of Leu 188 and Ala 189 residue (compound 15); between carbonyl group and $\mathrm{NH}$ of Leu 188 residues (compound 16) in the active site of MMP-9 protein, respectively. The hydroxamate group of all the docked compounds showed appreciable metal interaction with $\mathrm{Zn}^{2+}$ of the MMP-9 protein. In conclusion, the data strongly indicated that the selected compound 16 inhibit tumor invasion and migration by repressing MMP-2 and MMP-9 at protein and mRNA levels. Further, by directly interacting with active site residues to inhibit MMP-2 and MMP-9 activities. This study provides vital evidence about the anti-invasive potential of these prospecting candidates for developing into potential anticancer therapeutics, however further evidence should be conducted in in vivo studies to verify its application for clinical use in anti-metastatic effects on lung cancer cells. 


\section{Authors' contributions}

$V L$ proposed the subject, designed methods and experiments of the study and supervised the entire work. HD was involved in the commencement of the proposal, revised the manuscript decisively and had provided final version approval to be published. DR performed the experimental work, done data analysis, interpretation of results and prepared the draft of the manuscript. ASG was involved in silico studies and interpretation of results. VL modified the manuscript. All authors read and approved the final manuscript.

\section{Author details}

1 Department of Pharmaceutical Sciences, Maharshi Dayanand University, Rohtak, Haryana 124001, India. ${ }^{2}$ Department of Pharmaceutical Chemistry, JCDM College of Pharmacy, Sirsa, Haryana 125055, India. ${ }^{3}$ Chitkara College of Pharmacy, Chitkara University, Rajpura, Patiala, Punjab 140401, India.

\section{Acknowledgements}

Authors are thankful to Department of Pharmaceutical Sciences, Maharshi Dayanand University, Rohtak and Jan Nayak Ch. Devi Lal Memorial College of Pharmacy Sirsa for providing the facilities and uninterrupted support to carry out the work.

\section{Competing interests}

Authors declare that they have no competing interests and the authors alone are responsible for the content and writing of the paper

\section{Ethics approval and consent to participate} Not applicable.

\section{Publisher's Note}

Springer Nature remains neutral with regard to jurisdictional claims in published maps and institutional affiliations.

Received: 1 March 2018 Accepted: 13 April 2018

Published online: 20 April 2018

\section{References}

1. Babine RE, Bender SL, Kazeminejad B (1997) Molecular recognition of protein-ligand complexes: application to drug design. Chem Rev 97:1359-1472

2. Blagosklonny MV, Fojo T (1999) Molecular effects of paclitaxel: myths and reality (a critical review). Int J Cancer 83(2):151-156

3. Bremnes RM, Dønnem T, Al-Saad S, Al-Shibli K, Andersen S, Sirera R et al (2011) The role of tumor stroma in cancer progressionand prognosis: emphasis on carcinoma-associated fibroblastsand non-small cell lung cancer. J Thorac Oncol 6:209-217

4. Burzynski SR, Musial L (2002) Synthesis of 4-phenylbutyric acid, US Patent 2002-6372938, April 16; 2002

5. Cathcart J, Pulkoski-Gross A, Cao J (2015) Targeting matrix metalloproteinases in cancer: bringing new life to old ideas. Genes Dis 2:26-34

6. Davidson M, Smyth EC, Cunningham D (2016) Clinical role of ramucirumab alone or in combination with paclitaxel for gastric and gastroesophageal junction adenocarcinoma. OncoTargets Ther 9:4539

7. Djerassi C (1948) Brominations with N-bromosuccinimide and related compounds; the Wohl-Ziegler reaction. Chem Rev 43:271-317

8. Furniss BS, Hannaford AJ, Smith PWG, Tatchell R (1989) Vogel's textbook of practical organic chemistry. Longman Scientific \& Technical, London

9. Georgiadis MS, Russell EK, Gazdar AF, Johnson BE (1997) Paclitaxel cytotoxicity against human lung cancer cell lines increases with prolonged exposure durations. Clin Cancer Res 3(3):449-454

10. Gupta SP (ed) (2012) Matrix metalloproteinase inhibitors: specificity of binding and structure-activity relationships. Berlin, Springer Science \& Business Media, p 57

11. Ju Y, Varma RS (2006) Aqueous N-heterocyclization of primary amines and hydrazines with dihalides: microwave-assisted syntheses of $\mathrm{N}$-azacycloalkanes, isoindole, pyrazole, pyrazolidine, and phthalazine derivatives. J Org Chem 71:135-141
12. Kiyama R, Tamura Y, Watanabe F, Tsuzuki H, Ohtani M, Yodo M (1999) Homology modeling of gelatinase catalytic domains and docking simulations of novel sulfonamide inhibitors. J Med Chem 42:1723-1738

13. Lee MM, Chen YY, Liu PY, Hsu S, Sheu MJ (2015) Pipoxolan inhibits CL1-5 lung cancer cells migration and invasion through inhibition of MMP-9 and MMP-2. Chem Biol Interact 236:19-30

14. Levin JI, DiJosephJF Killar LM, Sung A, Walter T, Sharr MA et al (1998) The synthesis and biological activity of a novel series of diazepine MMP inhibitors. BioorgMed Chem Lett 8(19):2657-2662

15. Liebmann JE, Cook JA, Lipschultz C, Teague D, Fisher J, Mitchell JB (1993) Cytotoxic studies of paclitaxel $\left(\operatorname{TaxO}^{\circledR}{ }^{\circledR}\right)$ in human tumour cell lines. $\mathrm{Br} J$ Cancer 68(6):1104

16. Merchant N, Nagaraju GP, Rajitha B, Lammata S, Jella KK, Buchwald ZS et al (2017) Matrix metalloproteinases: their functional role in lung cancer Carcinogenesis 38(8):766-780

17. Morris GM, Huey R, Lindstrom W, Sanner MF, Belew RK, Goodsell DS et al (2009) AutoDock 4 and AutoDock Tools 4: automated docking with selective receptor flexibility. J Comput Chem 30(16):2785-2791

18. Nagase H, Robert V, Gillian M (2006) Structure and function of matrix metalloproteinases and TIMPs. Cardiovasc Res 69:562-573

19. Niu T, Zhang W, Huang D, Xu C, Wang H, Hu Y (2009) A powerful reagent for synthesis of Weinreb amides directly from carboxylic acids. Org Lett $11: 4474-4477$

20. O'Boyle NM, Banck M, James CA, Morley C, Vandermeersch T (2011) HutchisonGR. Open Babel: an open chemical toolbox. J Cheminform 3:33

21. Owonikoko TK, Ramalingam SS, Kanterewicz B, Balius TE, Belani CP, Hershberger PA (2010) Vorinostat increases carboplatin and paclitaxel activity in non-small cell lung cancer cells. Int J Cancer 126(3):743-755

22. Page-McCaw A, Ewald AJ, Werb Z (2007) Matrix metalloproteinases and the regulationof tissue remodelling. Nat Rev Mol Cell Biol 8:221-233

23. Pandey AK, Bhattacharya P, Shukla SC, Paul S, Patnaik R (2015) Resveratrol inhibits matrix metalloproteinases to attenuate neuronal damage in cerebral ischemia: a molecular docking study exploring possible neuroprotection. Neural Regen Res 10(4):568-575

24. Park WH, Kim SH, Kim CH (2005) A new matrix metalloproteinase-9 inhibitor 3,4-dihydroxycinnamic acid (caffeic acid) from methanol extract of Euonymus alatus: isolation and structure determination. Toxicol 207(3):383-390

25. Rajan P, Vedernikova I, Cos P, Berghe DV, Augustyns K, Haemers A (2001) Synthesis and evaluation of caffeic acid amides as antioxidants. Bioorg Med Chem Lett 11:215-217

26. Rathee D, Thanki M, Bhuva S, Anandjiwala S, Agrawal R (2013) Iridoid glycosides-Kutkin, Picroside I, and Kutkoside from Picrorrhiza kurroa Benth inhibits the invasion and migration of MCF-7 breast cancer cells through the down regulation of matrix metalloproteinases. Arab J Chem 6:49-58

27. Shapiro SD (1998) Matrix metalloproteinase degradation of extracellular matrix: biological consequences. Curr Opin Cell Biol 10:602-608

28. Shi ZH, Li NG, Shi QP, Tang H, Tan YP, Li W et al (2012) Design, synthesis and biological evaluation of caffeic acid amides as selective MMP-2 and MMP-9 inhibitors. Drug Dev Res 73:343-351

29. Shi ZH, Li NG, Shi QP, Tang H, Tang YP (2012) Design, synthesis, and preliminary evaluation of substituted cinnamic acid esters as selective matrix metalloproteinase inhibitors. Drug Dev Res 73:317-324

30. Tochowicz A, Maskos K, Huber R, Oltenfreiter R, Dive V, Yiotakis A et al (2007) Crystal structures of MMP-9 complexes with five inhibitors: contribution of the flexible Arg424 side-chain to selectivity. J Mol Biol 371:989-1006

31. Togawa D, Koshino T, Saito T, Takagi T, Machida J (1999) Highly activated matrix metalloproteinase- 2 secreted from clones of metastatic lung nodules of nude mice injected with human fibrosarcoma HT1080. Cancer Lett 146:25-33

32. Trott O, Olson AJ (2010) AutoDock Vina: improving the speed and accuracy of docking with a new scoring function, efficient optimization and multithreading. J Comput Chem 31(2):455-461

33. Vilela PD, de Oliveira JR, de Barros PP, Leão MV, de Oliveira LD, Jorge AO (2015) In vitro effect of caffeic acid phenethyl ester on matrix metalloproteinases (MMP-1 and MMP-9) and their inhibitor (TIMP-1) in lipopolysaccharide-activated human monocytes. Arch Oral Biol 60(9):1196-1202

34. Visse R, Nagase H (2003) Matrix metalloproteinases and tissue inhibitors of metalloproteinases: structure, function, and biochemistry. Circ Res 92:827-839 
35. Yu B, Tan L, Zheng R, Tan H, Zheng L (2016) Targeted delivery and controlled release of Paclitaxel for the treatment of lung cancer using single-walled carbon nanotubes. Mater Sci Eng C 68:579-584

36. Zhang J, Shen Y, Liu J, Wei D (2005) Antimetastatic effect of prodigiosin through inhibition of tumor invasion. Biochem Pharmacol 69(3):407-414

37. Zhang M, Altuwaiji S, Yeh S (2004) RRR-alpha-tocopheryl succinate inhibits human prostate cancer cell invasiveness. Oncogene 23:3080-3088
38. Zhao M, Lei C, Yang Y, Bu X, Ma H, Gong H et al (2015) Abraxane, the nanoparticle formulation of paclitaxel can induce drug resistance by upregulation of P-gp. PLoS ONE 10(7):e0131429

39. Zheng S, Chang Y, Hodges KB, Sun Y, Ma X, Xue Y et al (2010) Expression of KISS1 and MMP-9 in non-small cell lung cancer and their relations to metastasis and survival. Anticancer Res 30:713-718

\section{Submit your manuscript to a SpringerOpen ${ }^{\circ}$ journal and benefit from:}

- Convenient online submission

- Rigorous peer review

- Open access: articles freely available online

- High visibility within the field

- Retaining the copyright to your article

Submit your next manuscript at springeropen.com 